\title{
A search for pre- and proto-brown dwarfs in the dark cloud Barnard 30 with ALMA
}

\author{
N. Huélamo ${ }^{1}$, I. de Gregorio-Monsalvo ${ }^{2,3}$, A. Palau ${ }^{4}$, D. Barrado ${ }^{1}$, A. Bayo ${ }^{5,6}$, M. T. Ruiz ${ }^{7}$, L. Zapata ${ }^{4}$, H. Bouy ${ }^{1}$, \\ O. Morata ${ }^{8}$, M. Morales-Calderón ${ }^{1}$, C. Eiroa ${ }^{9}$, and F. Ménard ${ }^{10}$
}

${ }^{1}$ Dpto. Astrofísica, Centro de Astrobiología (INTA-CSIC); ESAC campus, Camino bajo del Castillo s/n, Urb. Villafranca del Castillo, 28692 Villanueva de la Cañada, Spain e-mail: nhuelamo@cab.inta-csic.es

2 European Southern Observatory, 3107 Alonso de Córdova, Vitacura, Santiago, Chile

3 Joint ALMA office, 3107 Alonso de Córdova, Vitacura, Casilla 19001, Santiago 19, Chile

${ }^{4}$ Instituto de Radioastronomía y Astrofísica, Universidad Nacional Autónoma de México, PO Box 3-72, 58090 Morelia, Michoacán, México

5 Departamento de Física y Astronomía, Facultad de Ciencias, Universidad de Valparaíso, Av. Gran Bretaña 1111, 5030 Casilla, Valparaíso, Chile

${ }^{6}$ ICM nucleus on protoplanetary disks, Universidad de Valparaíso, Av. Gran Bretaña 1111, Valparaíso, Chile

7 Dpto. de Astronomía, Universidad de Chile, 1515 Camino del Observatorio, Santiago, Chile

8 Institute of Astronomy and Astrophysics, Academia Sinica, 11F of Astronomy-Mathematics Building, AS/NTU. No. 1, Sec. 4, Roosevelt Rd, Taipei 10617, Taiwan

9 Unidad Asociada Astro-UAM, UAM, Unidad Asociada CSIC, Universidad Autónoma de Madrid, Ctra. Colmenar km 15, 28049 Madrid, Spain

10 Institut de Planétologie et d'Astrophysique de Grenoble, UMR 5274, BP 53, 38041 Grenoble, Cedex 9, France

Received 14 March 2016 / Accepted 21 September 2016

\begin{abstract}
Context. The origin of brown dwarfs is still under debate. While some models predict a star-like formation scenario, others invoke a substellar mass embryo ejection, a stellar disk fragmentation, or the photo-evaporation of an external core due to the presence of massive stars.

Aims. The aim of our work is to characterize the youngest and lowest mass population of the dark cloud Barnard 30, a region within the Lambda Orionis star-forming region. In particular, we aim to identify proto-brown dwarfs and study the mechanism of their formation.

Methods. We obtained ALMA continuum observations at $880 \mu \mathrm{m}$ of 30 sub-mm cores previously identified with APEX/LABOCA at $870 \mu \mathrm{m}$. We have complemented part of the ALMA data with sub-mm APEX/SABOCA observations at $350 \mu \mathrm{m}$, and with multiwavelength ancillary observations from the optical to the far-infrared (e.g., Spitzer, CAHA/O2000, WISE, INT/WFC).

Results. We report the detection of five (out of 30) spatially unresolved sources with ALMA, with estimated masses between 0.9

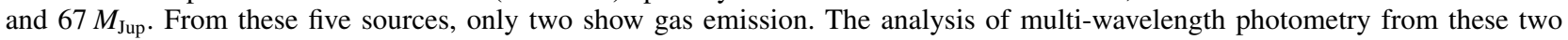
objects, namely B30-LB14 and B30-LB19, is consistent with one Class II- and one Class I low-mass stellar object, respectively. The gas emission is consistent with a rotating disk in the case of B30-LB14, and with an oblate rotating envelope with infall signatures in the case of LB19. The remaining three ALMA detections do not have infrared counterparts and can be classified as either deeply embedded objects or as starless cores if B30 members. In the former case, two of them (LB08 and LB31) show internal luminosity upper limits consistent with Very Low Luminosity objects, while we do not have enough information for LB10. In the starless core scenario, and taking into account the estimated masses from ALMA and the APEX/LABOCA cores, we estimate final masses for the central objects in the substellar domain, so they could be classified as pre-BD core candidates. According to the turbulent fragmentation scenario, the three ALMA pre-BD core candidates should be gravitationally stable based on APEX/LABOCA data. However, this result is not consistent with the presence of compact sources inside the cores, indicative of on-going collapse. As an alternative scenario we propose that these cores could be the result of on-going gravitational contraction. Indeed, we have verified that their estimated masses are consistent with the ones expected within an ALMA beam for a $r^{-2}$ density profile, which is typical for a collapsing core.

Conclusions. ALMA observations have allowed us to detect very low-mass compact sources within three APEX/LABOCA cores. Future observations will help us to unveil their true nature.
\end{abstract}

Key words. brown dwarfs - stars: formation - techniques: interferometric - radio continuum: general

\section{Introduction}

Brown dwarfs (BDs) are the bridge between low-mass stars and Jupiter-like planets. Although abundant in star forming regions (e.g., White \& Basri 2003; Barrado y Navascués et al. 2004; Caballero et al. 2007; Bouy et al. 2007; Bayo et al. 2011;
Alves de Oliveira et al. 2012; Scholz et al. 2013; Mužić et al. 2015), and the field (e.g., Delfosse et al. 1997; Delorme et al. 2008; Mainzer et al. 2011), their formation mechanism is still under debate. In low-mass star-forming regions, which typically form objects in groups or loose associations, the main brown dwarf formation mechanisms are turbulent fragmentation 
(Padoan \& Nordlund 2004; Hennebelle \& Chabrier 2008) and ejection from multiple protostellar systems and/or fragmented disks (Reipurth \& Clarke 2001; Bate et al. 2002; Matzner \& Levin 2005; Whitworth \& Stamatellos 2006). However, in the surroundings of high-mass stars, where objects typically form in a more tightly packed manner, there are additional mechanisms, namely photo-evaporation of cores near massive stars (e.g., Hester et al. 1996; Whitworth \& Zinnecker 2004) that have already been observed in young clusters (see e.g., Bouy et al. 2009; Hodapp et al. 2009), and gravitational fragmentation of dense filaments formed in a nascent cluster (e.g., Bonnell et al. 2008; Bate 2012).

The different BD formation scenarios can be tested by studying the earliest phases in the BD formation, when BDs are still embedded in the parental cloud, the so-called proto-BDs. The study of the most embedded and youngest BDs requires observations in the $\mathrm{mm} / \mathrm{sub}-\mathrm{mm}$ regime, where they emit the bulk of their energy as they are dominated by cold envelopes (e.g., Bourke et al. 2006; Lee et al. 2013; Palau et al. 2014).

Several studies have tried to unveil the youngest populations of BDs. The so-called very low luminosty objects (VeLLOs, e.g., Young et al. 2004; di Francesco et al. 2007; Dunham et al. 2008; Lee et al. 2013), which are characterized by internal luminosities $\leq 0.1 L_{\odot}$, were identified as potential proto-BDs. The so-called pre-brown dwarf (pre-BD) cores are also potential proto-BDs. They are cores with no infrared/optical counterparts that could be gravitationally bound but have very low masses (near substellar) and thus they will not be able to form a stellar object even if accreting all the reservoir of mass within the core. Good examples of pre-BD cores can be found in e.g. Palau et al. (2012) or André et al. (2012). Up to now, several objects have been identified as VeLLOs whose properties are consistent with protoBDs (see e.g., Young et al. 2004; Bourke et al. 2006; Lee et al. 2009; Barrado et al. 2009; Palau et al. 2012, 2014; Morata et al. 2015). However, the samples of proto-BD candidates are still very small.

In order to shed light on the BD formation process, in 2007 we started a project to study the low mass population of the dark cloud Barnard 30 (B30). B30 is located at the rim of the Lambda Orionis star-forming region (LOSFR, Murdin \& Penston 1977), a complex structure at a distance of $\sim 400$ pc (Perryman et al. 1997; Dolan \& Mathieu 1999), which gives shape to the head of Orion. The central part of the region is occupied by Collinder 69 , a $\sim 5 \mathrm{Myr}$ cluster (e.g., Dolan \& Mathieu 1999; Barrado y Navascués \& Jayawardhana 2004; Barrado y Navascués et al. 2007; Bayo et al. 2011) that harbours the O8 III star $\lambda$ Ori, while B30 is located $\sim 2.5$ degrees northwest from this massive star (see Fig. 1). The B30 stellar association was first identified by Duerr et al. (1982) and confirmed by Gomez \& Lada (1998). Later, the stellar population was studied by Dolan \& Mathieu (1999, 2001, 2002) who built a census of pre-main sequence (PMS) stars in the region. Using mid-IR data from the IRAC instrument onboard the Spitzer telescope, together with deep optical and near-IR data, Morales-Calderón (2008) was able to characterize the young low-mass population of the cloud, and to identify new candidate members with masses between 1.1 and $0.02 M_{\odot}$ reaching the substellar mass regime. Finally, Bayo (2009) built the census of $\sim 70$ spectroscopically confirmed members (including 15 new members), derived large accretion rates and accretion fractions, and calculated an optimal age estimation of between one and three Myr given the large dispersion in the HR diagram.

To further unveil the youngest population of B30, Barrado et al. (2016, BGH16, hereafter) analyzed APEX/
LABOCA $870 \mu \mathrm{m}$ deep observations (average rms of $7 \mathrm{mJy}$ ) of a region of $\sim 0.5^{\circ} \times 0.5^{\circ}$ within the cloud, and reported the detection of 34 sub-mm sources. They found IR counterparts for 15 of them within $5^{\prime \prime}$ from the LABOCA peak intensity coordinates, with nine being good substellar candidates. Thirteen objects did not show IR counterparts within $5^{\prime \prime}$, but showed several counterparts between 5 and 27 arcsec (the LABOCA image resolution) that might be responsible for the sub-mm emission. All of them (if B30 members) might also be substellar. Unfortunately, the low spatial resolution of the LABOCA map ( 27 arcsec) did not allow them to assign unambiguous infrared counterparts to most of the sub-mm sources. Finally, they also reported six potential starless cores, that might be pre-BD core candidates according to their estimated masses. As a result of their work, BGH16 built a census of proto- and pre-BD candidates in B30. We note that, recently, Liu et al. (2016) have also presented a study of cold cores in the $\mathrm{B} 30$ region, reporting the discovery of a Class 0 protostar and a proto-BD candidate, but in a different region of the cloud (at $\sim 27^{\prime}$ northwest from the APEX/LABOCA map obtained by BGH16).

In this work, we have further investigated the nature of the LABOCA sources identified by BGH16 through high angularresolution and high-sensitivity observations of B30 with the Atacama Large Millimeter Array (ALMA). The superb spatial resolution, great sensitivity, and positional accuracy of ALMA can help us to unambiguously assign the correct counterparts to the sub-mm sources, and to study their spectral energy distributions and dust content. We have complemented part of the ALMA observations with sub-mm data obtained with APEX/SABOCA at $350 \mu \mathrm{m}$. The observations are described in Sect. 2, while the data analysis is included in Sect. 3. The main results and conclusions are presented in Sects. 4 and 5.

\section{Observations}

\subsection{ALMA observations}

The ALMA observations were performed in 2012 on March 25th, December 3rd and December 12th at Band 7, as part of the ALMA Cycle 1 program 2012.0.00542.S. From the 34 detections reported in BGH16, we obtained individual pointings with a total field of view (FoV) of $\sim 18^{\prime \prime}$ for 30 of them (due to technical reasons, we did not observe the four sources LB01, LB32, LB33 and LB34). The central coordinates of each pointing correspond to the position of the pixel with maximum intensity of the $30 \mathrm{APEX} / \mathrm{LABOCA}$ detections. All the information about the 30 ALMA pointings is included in Table A.1.

A total of three data sets were collected, one for the northernmost region and two for the southernmost. We used 34 antennas (with $12 \mathrm{~m}$ diameter) in the northern region and 32 and 33 antennas in the south, accounting for $3.7 \mathrm{~h}$ of total integration time including overheads and calibration. Weather conditions were very good and stable, with an average precipitable water vapour of $0.9 \mathrm{~mm}$ (northern region) and $0.7-1.3 \mathrm{~mm}$ (southern region). The system temperature varied from 150 to $250 \mathrm{~K}$.

The correlator was set up to four spectral windows in dual polarization mode, centered at $345.8 \mathrm{GHz}, 347.8 \mathrm{GHz}$, $333.9 \mathrm{GHz}$, and $335.8 \mathrm{GHz}$ (average frequency of $340.8 \mathrm{GHz}$, or $880 \mu \mathrm{m})$. The effective bandwidth used per spectral window was $1875 \mathrm{MHz}$, providing a velocity resolution of $\sim 0.42 \mathrm{~km} \mathrm{~s}^{-1}$ after Hanning smoothing providing an average rms of $15 \mathrm{mJy} / \mathrm{beam}$.

The ALMA calibration includes simultaneous observations of the $183 \mathrm{GHz}$ water line with water vapour radiometers that measure the water column in the antenna beam, that is used 


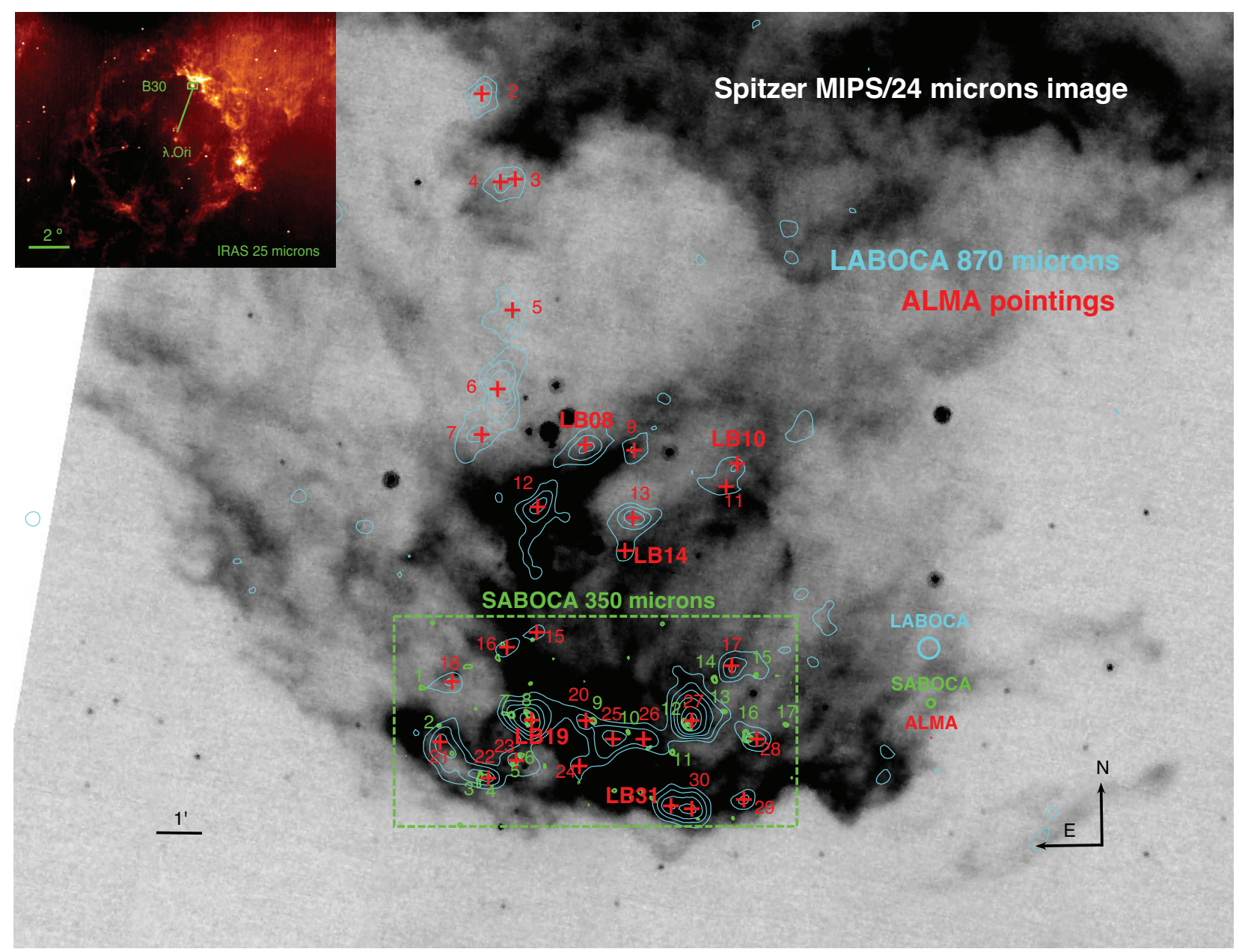

Fig. 1. Spitzer $24 \mu \mathrm{m}$ image of the B30 cloud. The displayed field of view is $\sim 23^{\prime} \times 18^{\prime}$. The cyan contours represent the APEX/LABOCA map at $870 \mu \mathrm{m}$ discussed in BGH16 (we show 3, 5, 7, 9, 12 and $15 \sigma$ levels). The red crosses show the ALMA pointing coordinates, and correspond to the pixels with maximum intensity of the LABOCA cores. The cores are numbered from 2 to 31 following BGH16. The five sources detected with ALMA are marked with red labels. The green contours (2.5, 3, 4 and $5 \sigma$ levels) represent the APEX/SABOCA data, and the green box the total field of view of the APEX/SABOCA map. We have numbered the 17 APEX/SABOCA sources that are detected above the $3 \sigma$ level. The beam sizes of each dataset are included on the right part of the image. Finally, the image at the top left corner shows the whole LORI region as seen by IRAS at $25 \mu \mathrm{m}$, and the green box represents the B30 area studied with APEX/LABOCA and ALMA.

to reduce the atmospheric phase noise. Amplitude calibration was carried out using Ganymede and J0510+180. The quasars J0532+0732 and J0607-0834 were used to calibrate the bandpass, and J0521+1638 and J0539+1433 for the complex gain fluctuations.

Data reduction was performed using version 4.2.2 of the Common Astronomy Software Applications package (CASA). Imaging of the calibrated visibilities was done using the task CLEAN. We applied self-calibration using the continuum data in sources B30S-13 (LB19) and B30N-18 (LB14). Briggs weighting with robust parameter equal 2 was used in all continuum and line images presented in this paper. The final images were corrected for the response of the primary beam and used to derive the physical parameters.

The average synthesized beam sizes of the continuum data in the north and south regions are $0 \prime \prime 93 \times 0$ " 50 (PA = 111 degrees) and $0 \prime \prime 95 \times 0 \prime \prime 48$ (PA $=-63.5$ degrees), respectively. The shortest baseline of the ALMA observations is $\sim 15 k \lambda$, while the upper end of the baseline range corresponds to $\sim 395 k \lambda$. Following Eq. (A5) from Palau et al. (2010), we estimate that the largest angular scale (LAS) detectable by the ALMA observations is $\sim 6^{\prime \prime}$.
We report the detection of five sources (out of 30 pointings) in our ALMA dataset. The five detected objects are spatially unresolved. Three sources are located in the northern region of B30, and two in the south, well within the ionization front facing the hot star $\lambda$ Ori (see Fig. 1). Their coordinates and fluxes are included in Table 1, while the ALMA continuum images are displayed in Fig. 2. The separation of the ALMA detections from the phase centers are included in Table 1. As can be seen, LB31 is very close to the phase center and shows an rms of $0.13 \mathrm{mJy}$. The other four sources are located at separations between 4.2 and 8.6 arcsec from the phase center and display a larger rms after correction from the response of the ALMA primary beam, which is $\sim 18 \operatorname{arcsec}$ (see Table 1). We note that these separations are well within the APEX/LABOCA beam (FWHM of 27.6") for all the ALMA detections. In the five cases, there are no other detected sources in the ALMA FoV. In the remaining 25 pointings no sources were detected within the whole ALMA FoV.

Although the ALMA observations were designed to detect continuum emission, the spectral setup was also selected to detect possible gas emission in the $\mathrm{CO}(3-2)$ transition at $345.8 \mathrm{GHz}$. The analysis of the spectral window centered at that frequency shows the presence of very extended gas emission, 

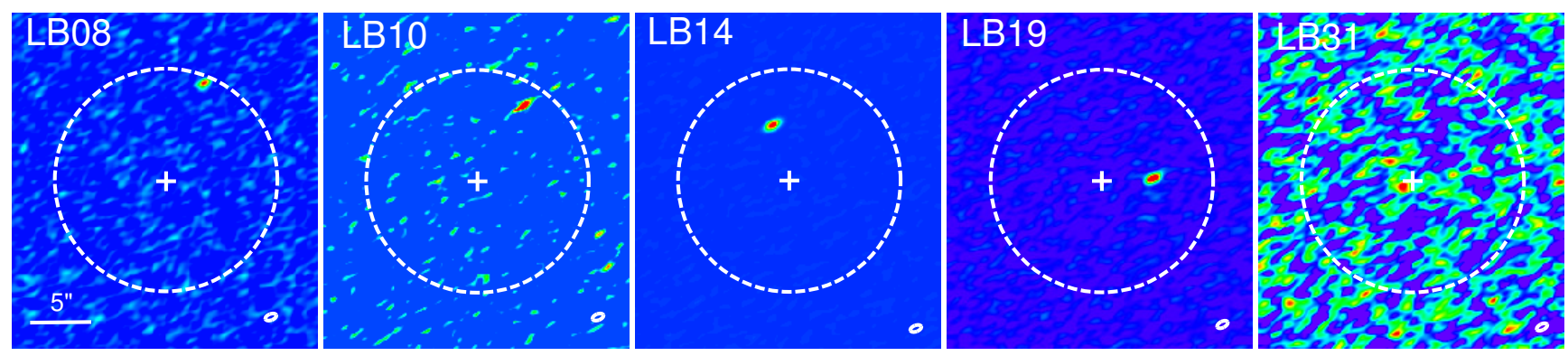

Fig. 2. The five sources detected at $880 \mu \mathrm{m}$ with ALMA in B30. North is up and east to the left. The ALMA beam size is displayed at the bottom right corner of each image. The white dashed circles represent the ALMA beam (18" diameter), and are centered at the pointing coordinates (phase centers) for each source.

Table 1. ALMA $880 \mu \mathrm{m}$ detections in the B30 region.

\begin{tabular}{|c|c|c|c|c|c|c|c|c|c|c|}
\hline $\begin{array}{l}\text { ALMA detec } \\
\text { RA(J2000) } \\
{[\mathrm{h} \mathrm{m} \mathrm{s}]}\end{array}$ & $\begin{array}{l}\text { ion coordinates } \\
\operatorname{Dec}(\mathrm{J} 2000) \\
{\left[\begin{array}{ll}{ }^{\circ},{ }^{\prime} & \end{array}\right]}\end{array}$ & $\begin{array}{l}\text { Separation from } \\
\text { phase center } \\
\text { [arcsec] }\end{array}$ & $\begin{array}{l}\text { LABOCA } \\
\text { source }^{a}\end{array}$ & $\begin{array}{l}S_{v} \\
{[\mathrm{mJy}]}\end{array}$ & $\begin{array}{l}\mathrm{rms} \\
{[\mathrm{mJy} / \text { beam }]}\end{array}$ & $\begin{array}{l}\text { Mass } \\
(\text { ALMA })^{* *} \\
{\left[M_{\text {Jup }}\right]}\end{array}$ & $\begin{array}{l}\text { Mass } \\
(\text { LABOCA })^{b, * *} \\
{\left[M_{\text {Jup }}\right]}\end{array}$ & $\begin{array}{l}\text { Missing } \\
\text { flux }^{c} \\
{[\%]}\end{array}$ & $\begin{array}{l}A_{\mathrm{v}}{ }^{d} \\
{[\mathrm{mag}]}\end{array}$ & $\begin{array}{l}\text { Tentative } \\
\text { nature }\end{array}$ \\
\hline $05: 31: 22.97$ & $+12: 11: 34.7$ & 8.7 & B30-LB08 & 5.70 & 0.26 & 9 & 106 & 87 & 2.5 & VeLLO/pre-BD \\
\hline 05:31:09.29 & $+12: 11: 08.8$ & 6.8 & B30-LB10 & 2.20 & 0.23 & 3 & 46 & 93 & 1.2 & pre-BD \\
\hline 05:31:19.46 & $+12: 09: 15.1$ & 4.5 & B30-LB14* & 44.0 & 0.25 & 67 & 51 & 0 & 2.0 & YSO \\
\hline $05: 31: 27.81$ & $+12: 05: 30.9$ & 4.2 & B30-LB19* & 10.7 & 0.20 & 16 & 182 & 86 & 2.8 & YSO \\
\hline 05:31:15.32 & $+12: 03: 38.2$ & 0.6 & B30-LB31 & 0.60 & 0.13 & 0.9 & 82 & 99 & 2.4 & pre-BD \\
\hline
\end{tabular}

Notes. ${ }^{(a)}$ LABOCA designation number (see BGH16); ${ }^{(b)}$ From BGH16; ${ }^{(c)}$ Percentage of missing flux between ALMA and LABOCA data; ${ }^{(d)}$ From the 2MASS extinction map; ${ }^{(*)}$ Sources with gas emission detected with ALMA; ${ }^{(*)}$ Derived for a dust temperature of 15 K.

mainly resolved out, in the primary beam of most of the positions observed in the southern region of B30, and very faint gas emission inside the primary beam of some of the positions surveyed in the northern part of the cloud. The $v_{\mathrm{lsr}}$ of the $\mathrm{CO}(3-2)$ cloud emission is $\sim 11 \mathrm{~km} \mathrm{~s}^{-1}$, consistent with one of the velocity components derived by Lang et al. $\left(2000,9.43 \mathrm{~km} \mathrm{~s}^{-1}\right)$ in the average spectrum of the region. The analysis of the ALMA data shows that we have detected gas emission in only two sources that are also detected in the continuum (see Table 1). They are discussed in detail in Sect. 3.

Finally, we have estimated the relative positional accuracy of the observations using Eq. (1) from Reid et al. (1988). Considering the beam major axis $\left(\sim 0.9^{\prime \prime}\right)$, we derive relative positional accuracies between $0.2^{\prime \prime}$ for SNR of 4.6 (LB31) and $\sim 5$ mas for a SNR of 176 (LB14). The absolute position accuracy is smaller than the synthesized beam width.

\subsection{SABOCA observations}

We performed observations in continuum at $350 \mu \mathrm{m}$ with the Submillimetre APEX Bolometer CAmera (SABOCA) installed at the Atacama Pathfinder EXperiment (APEX ${ }^{1}$ ) telescope. SABOCA is a 39-channel bolometer array with a $1.5^{\prime}$ field-ofview and a 7.8" full-width at half-maximum (FWHM) beam per bolometer channel. The data were obtained under program $\mathrm{C}$ 087.F-0011A-2011, between July 27th and 29th, 2011. Weather conditions were excellent with pwv $\sim 0.4-0.7 \mathrm{~mm}$ (average of $0.45 \mathrm{~mm}$ ). Opacity at the zenith was calculated using skydips, yielding zenith opacity values between 0.9 to 1.4. Pointing measurements were taken regularly. The pointing uncertainly is estimated to be approximately $2^{\prime \prime}$. The focus was verified at the

\footnotetext{
1 This work is partially based on observations with the APEX telescope. APEX is a collaboration between the Max-Plank-Institute fur Radioastronomie, the European Southern Observatory, and the Onsala Space Observatory.
}

beginning of each observation. Flux calibration was performed using Mars and Uranus as primary calibrators, and HL Tau, V883 Ori, VY CMa and CRL618 as secondary calibrators. The absolute flux calibration uncertainty is estimated to be $\sim 30 \%$.

Our map was centered at RA $=05: 31: 20.5$, Dec $=+12: 05: 45$, comprising a region of $\sim 8^{\prime \prime} \times 4^{\prime \prime}$. For processing the data we used the Bolometer Array Analysis Software $\left(\mathrm{BOA}^{2}\right)$ and $\mathrm{CRUSH}^{3}$ (see Kovács 2008) software packages. Data reduction processing included flat-fielding, calibration, opacity correction, correlated noise removal and de-spiking. Every scan was visually inspected in order to identify corrupted data. We used and optimized data processing to detect faint point-like sources (options -faint -deep in CRUSH). The final image was smoothed to a resolution of 10.6 arcsec. The total on-source observing time was $4.5 \mathrm{~h}$ and the rms reached was $\sim 50 \mathrm{mJy}$ at the central areas of the map.

We detected a total of 17 sources in the SABOCA map (see Figure 1) with SNR between 3.0 and 4.6. Their coordinates correspond to the pixel of maximum emission (peak intensity), and are provided in Table 2 together with the measured flux densities. All detections are point-like sources.

\subsection{Complementary data}

In addition to the ALMA and SABOCA observations presented here, we used ancillary data of B30 to complement our observations. The data are fully described in BGH16. Briefly, we gathered deep, multi-wavelength observations of B30 from the optical to the far-IR regime. The optical data were obtained with the Wide Field Camera (WFC) at the Isaac Newton Telescope in La Palma in two filters, $r$ and $i$ (INT/WFC), while deep near-IR data were collected with Omega 2000 at the Calar Alto Observatory (CAHA/O2000). The mid-IR data were obtained with the

\footnotetext{
2 http://www . apex-telescope.org/bolometer/laboca/boa/ http://www. submm. caltech.edu/sharc/crush/
} 
Table 2. SABOCA $350 \mu \mathrm{m}$ detections in the southern region of B30.

\begin{tabular}{|c|c|c|c|c|c|c|c|c|}
\hline $\begin{array}{l}\text { SABOCA } \\
\text { source }\end{array}$ & $\begin{array}{l}\mathrm{RA} \\
{[\mathrm{h} \mathrm{m} \mathrm{s}]}\end{array}$ & $\begin{array}{l}\text { Dec } \\
{\left[\begin{array}{lll}{ }^{\circ} & \prime \prime\end{array}\right]}\end{array}$ & $\begin{array}{l}\text { LABOCA } \\
\text { source }^{a}\end{array}$ & $\begin{array}{l}\text { Peak } \\
\text { intensity } \\
{[\mathrm{Jy}]}\end{array}$ & $\begin{array}{l}\mathrm{rms} \\
\text { [Jy/beam] }\end{array}$ & $S N R$ & $\begin{array}{l}A_{\mathrm{v}}^{b} \\
{[\mathrm{mag}]}\end{array}$ & $\begin{array}{l}\text { IR } \\
\text { counterparts? }\end{array}$ \\
\hline B30-SB01 & $05: 31: 37.68$ & 12:06:13.7 & & 0.16 & 0.05 & 3.2 & 2.3 & NO \\
\hline B30-SB02 & $05: 31: 36.09$ & $12: 05: 24.2$ & & 0.14 & 0.04 & 3.5 & 3.6 & YES, $3, ? ?$ \\
\hline B30-SB03 & $05: 31: 32.41$ & $12: 04: 16.7$ & B30-LB22 & 0.16 & 0.05 & 3.2 & 2.8 & YES, LB22f, NOT MEMBER \\
\hline B30-SB04 & $05: 31: 31.75$ & $12: 04: 18.6$ & B30-LB22 & 0.14 & 0.04 & 3.5 & 2.8 & YES, LB22a, MEMBER \\
\hline B30-SB05 & $05: 31: 29.47$ & $12: 04: 34.7$ & B30-LB23 & 0.15 & 0.05 & 3.0 & 3.0 & YES, LB23a, MEMBER \\
\hline B30-SB06 & $05: 31: 28.82$ & $12: 04: 44.8$ & B30-LB23 & 0.14 & 0.04 & 3.5 & 3.1 & $\mathrm{NO}$ \\
\hline B30-SB07 & $05: 31: 29.57$ & $12: 05: 37.7$ & & 0.17 & 0.05 & 3.4 & 3.2 & YES, , MEMBER \\
\hline B30-SB08 & $05: 31: 27.94$ & $12: 05: 31.3$ & B30-LB19 & 0.23 & 0.05 & 4.6 & 2.7 & YES, LB19a, MEMBER \\
\hline B30-SB09 & $05: 31: 22.29$ & $12: 05: 30.7$ & B30-LB20 & 0.16 & 0.05 & 3.2 & 1.7 & NO \\
\hline B30-SB 10 & $05: 31: 19.12$ & $12: 05: 15.0$ & & 0.15 & 0.05 & 3.0 & 2.4 & NO \\
\hline B30-SB11 & $05: 31: 15.20$ & $12: 04: 49.3$ & & 0.16 & 0.05 & 3.2 & 3.4 & YES, 2, ?? \\
\hline B30-SB12 & $05: 31: 13.98$ & $12: 05: 25.3$ & B30-LB27 & 0.19 & 0.05 & 3.8 & 4.1 & NO \\
\hline B30-SB 13 & $05: 31: 10.55$ & $12: 05: 43.3$ & & 0.15 & 0.05 & 3.0 & 4.2 & YES, $1, ? ?$ \\
\hline B30-SB14 & $05: 31: 11.39$ & $12: 06: 24.2$ & & 0.16 & 0.05 & 3.2 & 3.9 & YES, $1, ? ?$ \\
\hline B30-SB 15 & $05: 31: 07.69$ & $12: 06: 30.9$ & & 0.16 & 0.05 & 3.2 & 2.6 & YES, $1, ? ?$ \\
\hline B30-SB16 & $05: 31: 08.45$ & $12: 05: 07.3$ & B30-LB28 & 0.22 & 0.05 & 4.4 & 3.8 & YES, LB28a, MEMBER \\
\hline B30-SB17 & $05: 31: 04.98$ & $12: 05: 25.3$ & & 0.22 & 0.07 & 3.1 & 1.8 & $\mathrm{NO}$ \\
\hline
\end{tabular}

Notes. ${ }^{(a)}$ LABOCA designation number (from BGH16); ${ }^{(b)}$ From the 2MASS extinction map; (c) In this column we specify if the SABOCA source shows counterparts in the IR, the number of counterparts (or its name if included in BGH16), and their nature: member/not member of B30 (according to BGH16), or unknown (??).

Spitzer space telescope with both the IRAC and MIPS instruments, covering a wavelength range from $3.6 \mu \mathrm{m}$ to $70 \mu \mathrm{m}$. We also complemented our database with archival observations from the infrared observatory WISE, far-IR data from the AKARI mission, and the Planck Catalog of compact sources (Release 1). Finally, we also considered the $870 \mu \mathrm{m}$ LABOCA data included in BGH16. The pointing errors, completeness and limiting magnitudes of all these data (except Planck) are fully described in BGH16.

We also used an extinction map of the B30 cloud based on 2MASS $J$-band data and derived using the star-count method presented in Cambresy et al. (1997). We estimated $A_{v}$ from $A_{J}$ using the relations by Fitzpatrick (1999). The values of $A_{v}$ at the position of the ALMA and SABOCA detections are provided in Tables 1 and 2, respectively. We note that the average spatial resolution of the extinction map is 1.5 , and the typical uncertainties are of $\sim 0.1 \mathrm{mag}$.

\section{Data analysis}

\subsection{ALMA detections: continuum and gas emission}

As mentioned above, we detected five sources with ALMA, all of them spatially unresolved. Two of these sources, LB14 and LB19, also show gas emission.

If we assume that the dust emission is optically thin, we can estimate the total mass (gas and dust) from thermal continuum emission following the expression:

$M=\frac{S_{v} D^{2}}{B_{v}\left(T_{\mathrm{d}}\right) \kappa_{v}}$,

where $S_{v}$ is the flux density, $D$ is the distance to the source, $B_{v}\left(T_{\mathrm{d}}\right)$ is the Planck function at the dust temperature $T_{\mathrm{d}}$, and $\kappa_{v}$ is the absorption coefficient per unit of total mass density. We

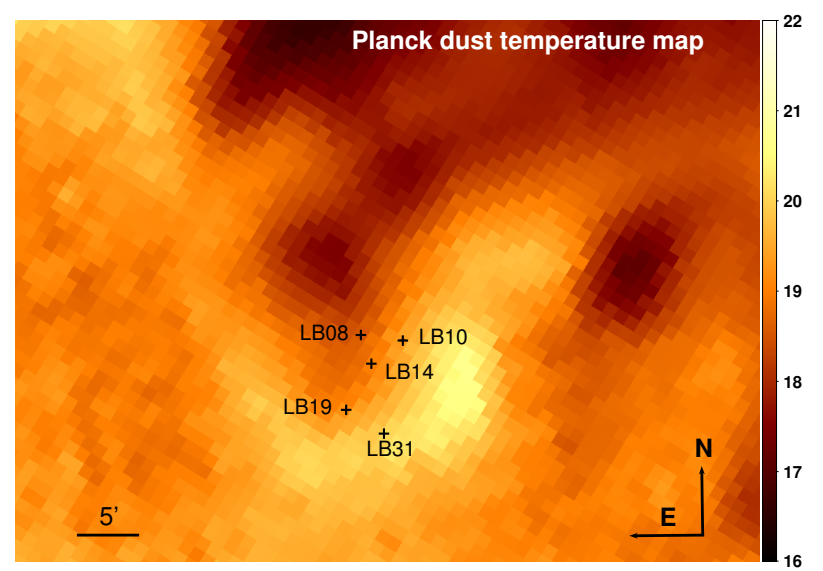

Fig. 3. Dust temperature map of the $\mathrm{B} 30$ region $\left(\mathrm{FoV}\right.$ of $\left.\sim 1^{\circ} \times 0.7^{\circ}\right)$ from the Planck mission (Planck Collaboration XI 2014). The color bar displays the temperature values in $\mathrm{K}$. We have included the positions of the detected ALMA sources.

can rewrite Eq. (1) in this way:

$\left[\frac{M}{M_{\odot}}\right]=3.25 \frac{\mathrm{e}^{0.048 v / T_{\mathrm{d}}}-1}{v^{3} \kappa_{v}}\left[\frac{S_{v}}{\mathrm{Jy}}\right]\left[\frac{D^{2}}{\mathrm{pc}^{2}}\right]$.

The dust temperature maps generated by the Planck mission (Planck Collaboration XI 2014) show temperature values between 17 and $21 \mathrm{~K}$ in the B30 region (see Fig. 3). This temperature can be considered as an upper limit to the dust temperature of the starless cores of B30, where the temperature should decrease towards the core centers (for example, the starless core in the Pipe Nebula FeSt 1-457 presents a decrease in dust temperature from $\sim 19 \mathrm{~K}$ in the core outskirts down to $\sim 14 \mathrm{~K}$ in the core center, Forbrich et al. 2015). On the other hand, the Planck temperature should be considered as a lower 
limit to the dust temperature for cores already harbouring protostars. Therefore, we have selected a conservative dust temperature of $15 \mathrm{~K}$ to estimate the masses of the cores in B30. We have estimated the absorption coefficient for an average frequency of $340.8 \mathrm{GHz}(\sim 880 \mu \mathrm{m})$ interpolating from the tables of Ossenkopf \& Henning (1994) for the case of thin ice mantles and a density of $10^{6} \mathrm{~cm}^{-3}$. We obtained a $k_{v}$ of $0.01715 \mathrm{~cm}^{2} \mathrm{~g}^{-1}$ for a gas to dust mass ratio of 100. Finally, we have assumed a distance of $400 \mathrm{pc}$, and the ALMA flux densities included in Table 1. The estimated masses are included in Col. 7 of Table 1. They range from $\sim 0.9$ to $66 M_{\text {Jup }}$. We note that the uncertainty in the masses due to the opacity law and dust temperature is estimated to be a factor of four.

The comparison of the masses derived from ALMA data with those derived from APEX/LABOCA data shows much smaller values for the ALMA detections (see Cols. 7 and 8 in Table 1). We have therefore compared the fluxes from the two datasets and derived the percentage of flux that ALMA did not recover compared to a single-dish (LABOCA). This is provided in Col. 9 of Table 1. We conclude that for all the sources except LB14, ALMA is missing $>85 \%$ of the flux detected by LABOCA.

We looked for possible infrared/optical counterparts to the ALMA sources using a search radius of $1^{\prime \prime}$ (the ALMA beam) and found clear infrared counterparts for two out of five ALMA detections (see Fig. 4): LB14 and LB19. The counterparts are the sources named LB14a and LB19a in BGH16, and were detected within the inner $5^{\prime \prime}$ of the LABOCA beam. Their SEDs are included in Fig. 5.

Three ALMA sources, namely LB08, LB10 and LB31 did not show any counterpart within $1^{\prime \prime}$ radius. BGH16 reported infrared counterparts (namely LB08a and LB10a) within a 5" radius for the two LABOCA cores (LB08 and LB10) but the positional accuracy of ALMA (and the CAHA and Spitzer telescopes) is good enough to reject them as the $880 \mu \mathrm{m}$ sub-mm emitters. For these three objects we also searched for counterparts in the NASA/IPAC Extragalactic Database (NED), but did not find any object within a 5" radius of the ALMA position.

The individual ALMA detections are discussed in more detail in the following subsections. We begin by describing the sources with IR counterparts and end by describing those without them.

\subsubsection{LB14}

LB14 has a very bright counterpart at optical and infrared wavelengths, named \#a in BGH16, and classified as a Class II stellar member (see Fig. 4). The ALMA detection is clearly assigned to this infrared source, with a separation of $0 \prime 3$. We note that this object was already classified by Dolan \& Mathieu (2002) as a pre-Main Sequence (PMS) star.

We have compared the optical photometry of the target $(R=16.016 \pm 0.032, I=14.486 \pm 0.032 \mathrm{mag}$, Dolan \& Mathieu 2002 ), corrected by the visual extinction provided in Table 1 , with BT-SETTL evolutionary models (Allard et al. 2012). We obtained that the central star shows a mass of $\sim 0.3 M_{\odot}$ (or $\sim 0.5 M_{\odot}$ ) for an age of $1 \mathrm{Myr}$ (or $3 \mathrm{Myr}$ ).

The IRAC colors of LB14a, [3.6]-[4.5] $=0.56$ and [5.8]$[8.0]=0.80 \mathrm{mag}$, are consistent with a Class II object (Allen et al. 2004). The SED is displayed in Fig. 5 (left panel) showing a clear excess at long wavelengths. The object is not included in the field of APEX/SABOCA.

The source is point-like in both the APEX/LABOCA and ALMA data. In fact, the estimated sub-mm fluxes are very similar in the two datasets $(35 \pm 14 \mathrm{mJy}$ versus $44.0 \pm 0.5 \mathrm{mJy}$, assuming $2 \sigma$ errors), with estimated masses of $\sim 51 M_{\text {Jup }}$ and $\sim 67 M_{\mathrm{Jup}}$, respectively, and $0 \%$ of ALMA missing flux when compared to the APEX/LABOCA emission. Given the evolutionary stage of LB14, we expect that the estimated mass is mainly associated with a circumstellar disk. In fact, since this object is evolved in comparison with starless cores, the dust temperature should be higher than the assumed $15 \mathrm{~K}$. We estimate a disk mass of $43 M_{\text {Jup }}$ for $20 \mathrm{~K}$, or $24 M_{\text {Jup }}$ for $30 \mathrm{~K}$. The angular resolution of the ALMA data provides an upper limit to the disk radius of $<140 \mathrm{AU}$.

LB14 shows gas emission in the ALMA data. The $\mathrm{CO}(3-$ 2) emission is very compact, spatially unresolved, and centered at the position of the dust emission (see Fig. 6, left top panel). The gas emission is seen from 8.3 to $12.1 \mathrm{~km} \mathrm{~s}^{-1}$. The spectrum shows a double peak emission (Fig. 6, left bottom panel) suggesting a disk in rotation, with the red-shifted emission located to the southeast and the blue-shifted one to the northwest of the beam center. The spectrum is centered at a $V_{\mathrm{lsr}}$ of $10.4 \mathrm{~km} \mathrm{~s}^{-1}$, confirming this source as a member of B30.

\subsubsection{LB19}

In the case of LB19, the ALMA detection is associated with source \#a in BGH16 (see Fig. 4). The source is undetected in the optical images, but detected in the $J$-band with a brightness that increases towards longer wavelengths. The source has a clear spatially resolved counterpart in the Spitzer images. Its IRAC colors, [3.6]-[4.5] = 1.34, [5.8]-[8.0] = 1.37 are consistent with a Class I source (Allen et al. 2004).

In the APEX/LABOCA map, LB19 is detected as an extended source with a deconvolved angular size of $24^{\prime \prime}$. The target is also detected in the APEX/SABOCA map and looks slightly elongated. However, the low SNR of the $350 \mu \mathrm{m}$ data does not allow its confirmation as an extended source.

The SED of the object is displayed in the central panel of Fig. 5 and its shape resembles that of a Class I object. In fact, LB19 is associated with the IRAS source IRAS 05286+1203 $\left(\mathrm{RA}=82.8671\right.$, Dec $=+12.0899$, separation of $\left.0.2^{\prime \prime}\right)$ included in the near-IR survey of Class I protostars by Connelley et al. (2008). They estimated a $L_{\text {bol }}$ of $14.4 L_{\odot}$ considering only the emission from the four IRAS bands. The source has been detected with Spitzer/MIPS at $70 \mu \mathrm{m}$ and displays a significantly smaller flux than in IRAS $60 \mu \mathrm{m}(0.94 \mathrm{Jy}$ vs. $9.35 \mathrm{Jy})$, with the latter value displaying an IRAS quality flag of "2" (moderate quality). If we integrate the whole SED without considering the IRAS data-points, we obtain a $L_{\text {bol }}$ of $1.2 L_{\odot}$ and a temperature of $T_{\mathrm{bol}} \sim 235 \mathrm{~K}$, which is consistent with a low-mass protostar.

The estimated mass from the ALMA data is $\sim 16 M_{\text {Jup }}$, while the estimation from APEX/LABOCA data is $182 M_{\text {Jup }}$ considering all the integrated emission, or $116 M_{\mathrm{Jup}}$ if we only consider the peak intensity. Such a difference is probably related to the APEX/LABOCA measurement (peak intensity of $\sim 79 \mathrm{mJy}$ ) including a significant emission from the surrounding cloud.

LB19 is the second source with a gas detection. The $\mathrm{CO}(3-2)$ emission shows two components: one that is compact and spatially coincident with the continuum detection, and an extended component (baselines $<60 \mathrm{k} \lambda$ ) of $\sim 2400$ AU surrounding it (Fig. 6, top middle and right panels). Both compact and extended components show emission from 5.7 to $14.2 \mathrm{~km} \mathrm{~s}^{-1}$ and are centered at $10.4 \mathrm{~km} \mathrm{~s}^{-1}$, which confirms LB19 as a B30 member. The intensity-weighted velocity field of both compact and extended components (first-order moment) shows a velocity 
N. Huélamo et al.: A search for pre- and proto-brown dwarfs in the dark cloud Barnard 30 with ALMA
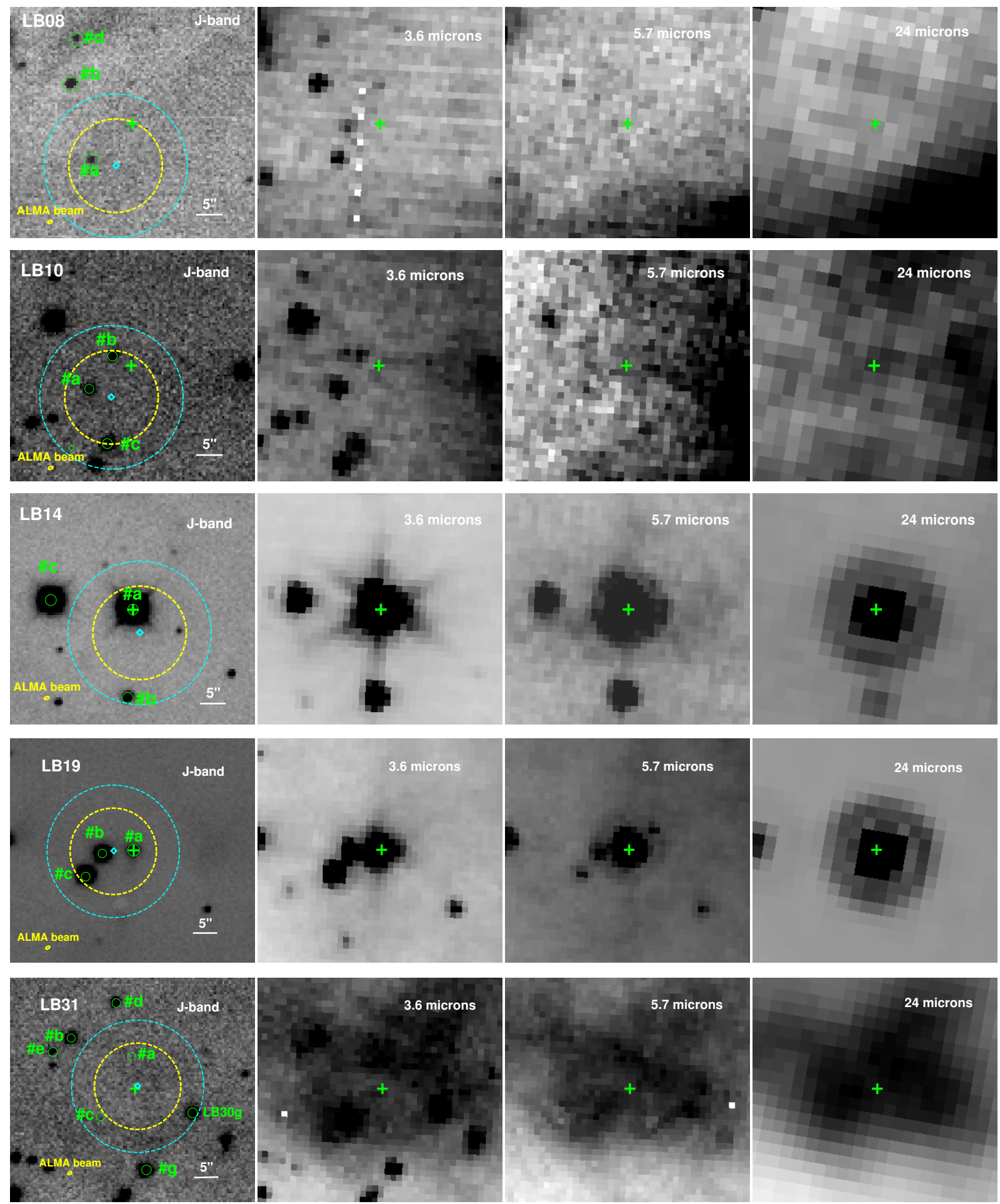

Fig. 4. Infrared (CAHA/O2000 and Spitzer/IRAC and MIPS) images centered on the ALMA detection coordinates. North is up and east to the left. We have included in all the $J$-band images (left panels) the position of the ALMA detections (green crosses), and the possible infrared counterparts to the APEX/LABOCA detections identified by BGH16 (green labels). The cyan diamonds represent the APEX/LABOCA peak intensity coordinates (ALMA phase center coordinates). We have also plotted the APEX/LABOCA beam of 13.8" radius (cyan dashed circle), the ALMA FOV of $\sim 18^{\prime \prime}$ diameter (yellow dashed circle), and the ALMA beam at the bottom left corner (yellow solid ellipse).

gradient with the most blueshifted and redshifted material at the tips of the gradient.

The spectrum of the compact component is asymmetric, with the blueshifted side stronger than the redshifted one (see Fig. 6, top middle panel). This might suggest infall is occurring at small scales $(\sim 400 \mathrm{AU})$ in this source.
The extended component is slightly elongated in the southeast northwest direction and shows also extended emission close to the cloud velocity $\left(\sim 11.5 \mathrm{~km} \mathrm{~s}^{-1}\right)$ in the southwest northeast direction. There is no presence of high velocity wings related to molecular outflows. The kinematical pattern might suggest that we are observing a rotating oblate envelope with probable 

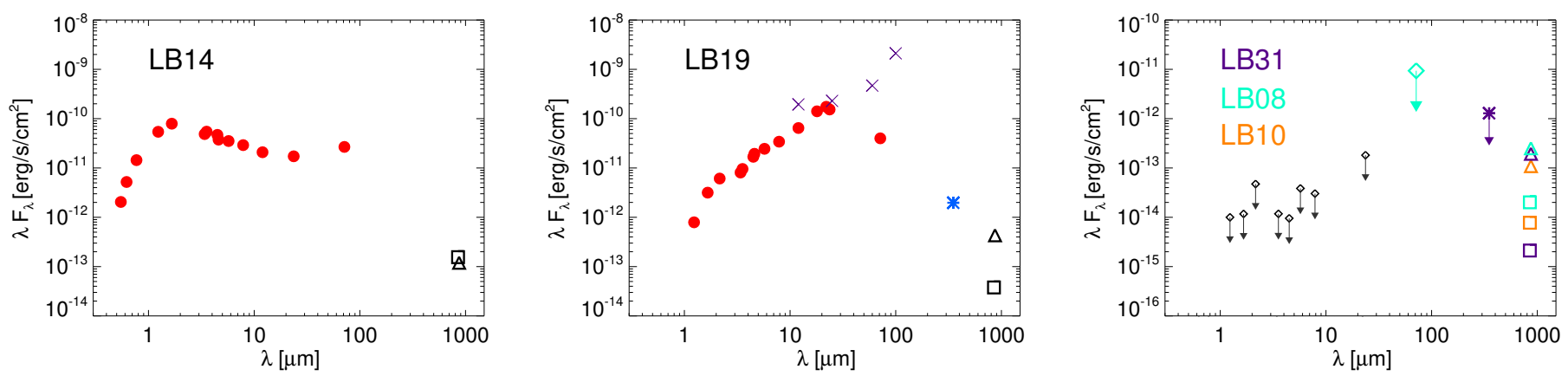

Fig. 5. Observed SEDs of the five ALMA detections. In all the panels, the open triangles and squares represent the APEX/LABOCA and ALMA fluxes, respectively, while the asterisks represent the APEX/SABOCA data. Left and middle panels: we show the complete SEDs of LB14 and LB19. The red circles represent optical to mid-IR data from all our catalogues whenever available (see Sect. 2.3). The purple crosses in the LB19 panel represent IRAS data. Right panel: three ALMA detections with no infrared counterparts: LB08, LB10 and LB31. The APEX/LABOCA and ALMA detections are color-coded for each source. We have included the limiting magnitudes (black diamonds) of our infrared data.
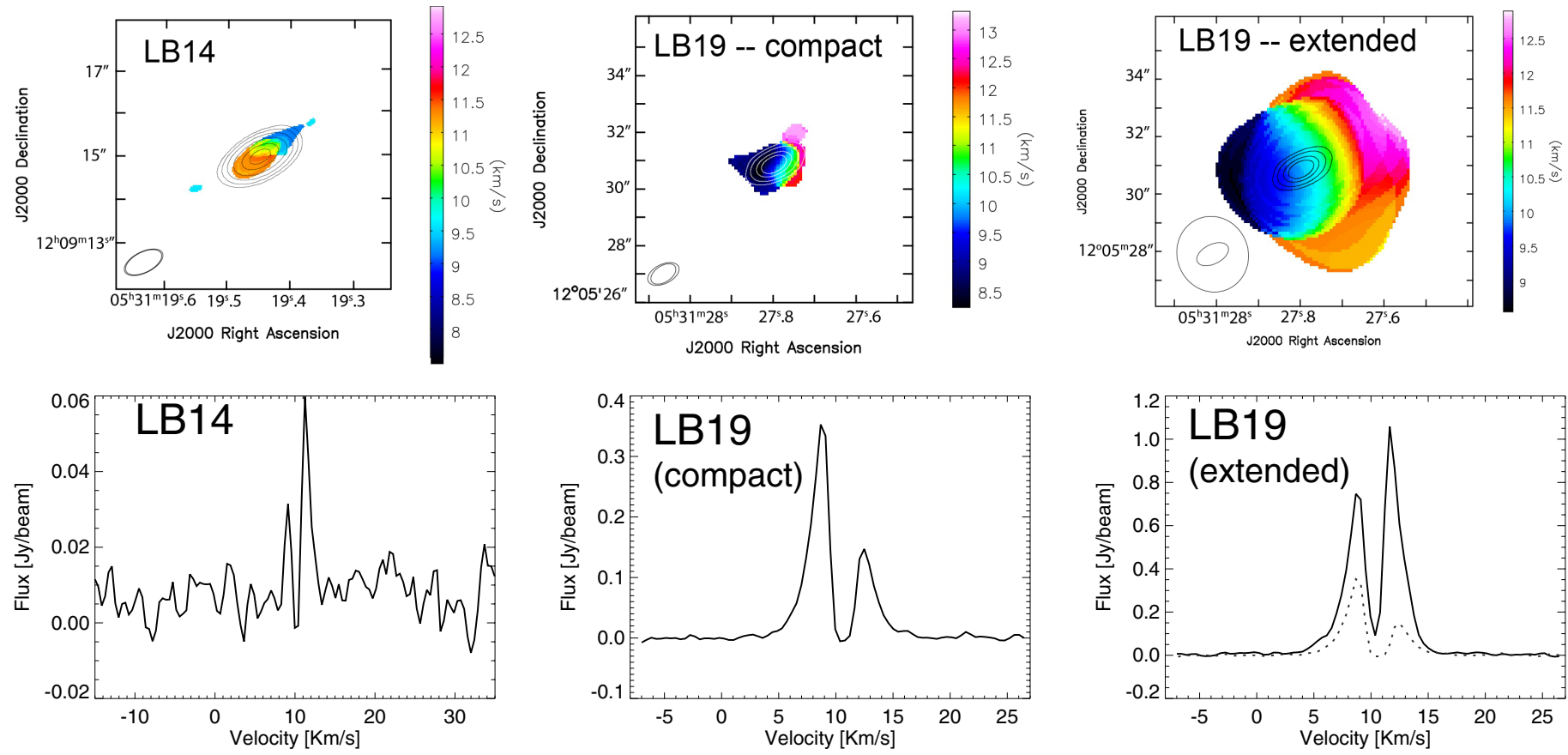

Fig. 6. Top panels: ALMA CO(3-2) intensity weighted velocity field (first order moment, color scale) with the ALMA 880 micron continuum emission over-plotted in contours. The left panel corresponds to LB14, while the middle and right panels correspond to the compact and extended emission of LB19, respectively. For LB14, contours are 5, 10, 20, 40, 80, and 120 times the rms noise of the map, $0.25 \mathrm{mJy} / \mathrm{beam}$. For LB19, contours are 5, 10, 20, and 30 times the rms noise of the map, $0.20 \mathrm{mJy} /$ beam. Ellipses at the bottom left represent the synthesized beam for the continuum and for the $\mathrm{CO}(3-2)$ maps. In the case of $\mathrm{LB} 19$, the beam for the $\mathrm{CO}(3-2)$ image corresponds to emission at $>60 \mathrm{~K} \lambda$ (central panel), and $<60 \mathrm{~K} \lambda$ (right panel). Bottom panels: $\mathrm{CO}(3-2)$ spectrum in the region of emission of LB14 (left), and in the compact (middle) and extended (right) regions of emission of LB19. In the last panel both the compact (dotted line) and extended emission (solid line) are superimposed for comparison.

infalling at $400 \mathrm{AU}$ scales near the central protostar. The extended component does not show a clear infalling pattern.

\subsubsection{LB08, LB10, and LB31}

LB08, 10, and 31 are the weakest sources detected with ALMA. There is no presence of gas emission at their positions, with an rms per channel of $13 \mathrm{mJy} / \mathrm{beam}, 11 \mathrm{mJy} / \mathrm{beam}$, and $18 \mathrm{mJy} /$ beam, respectively.

While LB08 and LB10 lie in the northern region of B30, LB31 is located in the southern part of the cloud, well within the ionization front seen in the $24 \mu \mathrm{m}$ image (see Fig. 1). While LB08 shows extended emission in the APEX/LABOCA map, LB08 and LB10 are detected as point-like sources. None of these ALMA detections show a clear counterpart in the infrared regime (Fig. 4).

LB08 shows a deconvolved angular size of $27^{\prime \prime}$ in the APEX/LABOCA data after fitting a Gaussian. BGH16 identified an infrared counterpart within the inner $5^{\prime \prime}$ of the LABOCA beam: LB08a (see Fig. 4). However, the ALMA absolute positional accuracy is good enough to conclude that this infrared source is unrelated with the ALMA detection. We note that there is a detection with AKARI/FIS at 65, 90, 140 and $160 \mu \mathrm{m}$ at a separation of 24" from the ALMA source (AKARI source name 0531239+121115). The AKARI/FIS PSF FWHM at the four different bands are 37, 39, 58 and 61 arcsec, respectively, with a positional accuracy of $\sim 8$ arcsec (Yamamura et al. 2009). The Spitzer/MIPS image at $70 \mu \mathrm{m}$ does not cover the whole field 

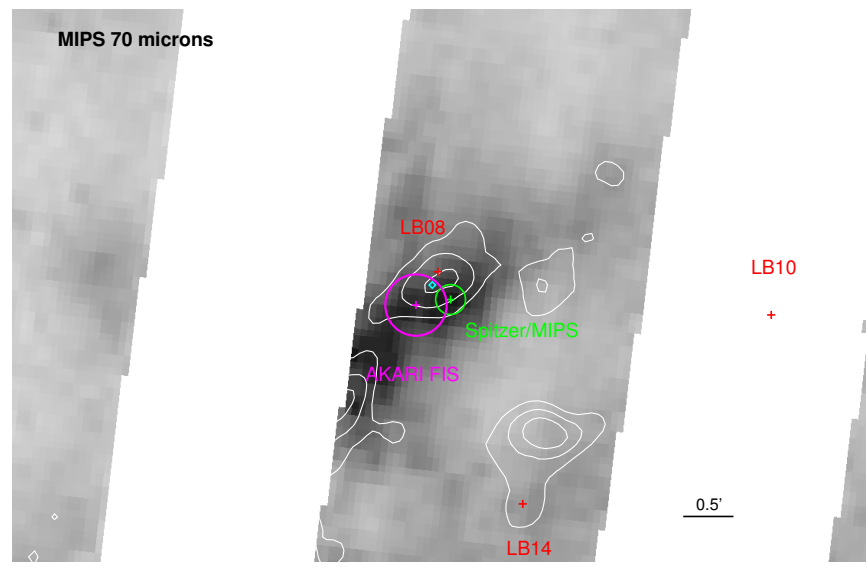

Fig. 7. Spitzer/MIPS $70 \mu \mathrm{m}$ sub-image centered on the ALMA source LB08 within B30. The white regions do not have data available. We overplotted the LABOCA contours (white), the ALMA detections (red), and the AKARI/FIS detection (magenta). The magenta circle represents the FWHM PSF of the AKARI FIS data at $65 \mu \mathrm{m}$ (37" diameter). The source LB08 (red cross) is within a bright filamentary structure detected at 70 microns and extending from the northwest to the southeast. The $\mathrm{MIPS} / 70 \mu \mathrm{m}$ peak emission (green cross) is located at $\sim 18 \operatorname{arcsec} \mathrm{SW}$ from the ALMA detection, and the green circle represents the FWHM of the PSF (18" diameter for MIPS).

of B30, and displays stripes without data every $3^{\prime}$ (see Fig. 7). However, the region of LB08 is included and its surroundings show bright extended emission close to the ALMA detection. The bulk of this emission (the MIPS peak intensity is at $18^{\prime \prime} \mathrm{SW}$ from the ALMA detection) is located more to the south, tracing a filament along the northwest-southeast direction (the MIPS PSF FWHM at 70 microns is of $\left.18^{\prime \prime}\right)$. In fact, the AKARI/FIS detection is at the center of this region and it probably includes most of the emission of the filamentary structure. We therefore conclude that AKARI detection seems not to be associated with the ALMA source but, alternatively, with the filament. On the other hand, the fact that the ALMA source is detected in this filament reinforces the hypothesis that it belongs to the $\mathrm{B} 30$ region and is not a background contaminant.

In the case of LB10, BGH16 found an infrared counterpart (source \#a) within 5 arcsec of the LABOCA beam (see Fig. 4). The ALMA data show that the detection is clearly unrelated to \#a and, in fact, is closer to source \#b, classified as a B30 nonmember by BGH16. The separation between the ALMA detection and source $\# \mathrm{~b}$ is of $\sim 4^{\prime \prime}$, therefore they seem unrelated. Inspection of the Spitzer/IRAC and MIPS images does not reveal any counterpart at the position of the ALMA source either.

Finally, LB31 is detected with ALMA with a SNR of 4.6 . BGH16 did not find any IR counterparts within 5 arcsec of the sub-mm LABOCA source, and we do not identify any counterpart at the position of the ALMA source. We only detect extended emission from the B30 cloud at $24 \mu \mathrm{m}$, since the object is well within the ionization front of the cloud (see Fig. 1 and a zoom of the LB31 region in Fig. 4). This is the only object (out of the three) that is included in the SABOCA map, but it is not detected at $350 \mu \mathrm{m}$.

Note that neither LB10 nor LB31 have $70 \mu \mathrm{m}$ MIPS data available, and they are not detected with AKARI. As mentioned above, neither of them appears to have a counterpart in the NED database.

\subsection{APEX/SABOCA detections}

We detected a total of 17 sources in the APEX/SABOCA map at $350 \mu \mathrm{m}$ (see Fig. 1). Eight out of the 17 seem to be associated with LABOCA cores: sources B30-SB03 to 06 are contained within the southern filament revealed in the LABOCA contour map. While SB03 and SB04 seem to be related to B30LB22, B30-SB05 and B30-SB06 are associated with B30-LB23 (Fig. 1). B30-SB08 is related to B30-LB19 (already discussed in Sect. 3.1.2). B30-SB09, at the center of the map, is at $\sim 9$ arcsec from B30-LB20. In the western part of the map we find B30SB12, at 10.5" from the bright core B30-LB27, and B30-SB16, at $11.5^{\prime \prime}$ from B30-LB28. The low SNR of the detections has prevented us from estimating the dust masses.

We looked for infrared counterparts to all the SABOCA sources in our Spitzer/IRAC and CAHA/O2000 catalogues. We used a diameter search of $10^{\prime \prime} .6$ which corresponds to the SABOCA beam. We also looked for possible counterparts with WISE $^{4}$ and AKARI. The finding charts are displayed in Fig. 8, and the SEDs of the detected counterparts in Fig. 9. For the eight sources that seem associated with LABOCA cores, we have refined the assignment of infrared counterparts from BGH16. For the SABOCA sources not associated with LABOCA cores, we can see that they all lie within the southern filament detected in the LABOCA map (excluding source 17, at the edge of the map), and are associated with some $870 \mu \mathrm{m}$ emission at a $\geq 3 \sigma$ level (sources 02, 07, 10, 13, and 15, see Fig. 1) or with a more marginal $(<3.0 \sigma)$ emission (sources 01,11 , and 14). For these sources we have represented the LABOCA peak emission at the SABOCA position in their SEDs.

We found infrared counterparts for 11 SABOCA sources. However, only one of them (LB19a) is detected close to the center of the SABOCA beam at a separation of 1.7 arcsec. For the ten remaining sources, the assignment of an IR counterpart is uncertain, but we describe our findings below for completeness (to facilitate the reading, we have omitted the prefix B30 in the APEX/SABOCA source names).

For $S B 02$ we detected three possible Spitzer/IRAC counterparts, all of them at separations between 4 " and $5^{\prime \prime}$. Sources \#a and \#c are also detected in the near-IR. SB02 has a single WISE counterpart at a separation of 1.3 arcsec (WISE J053136.15+120526.4). Given its smaller spatial resolution, we think that the WISE source might contain emission from the three sources detected by Spitzer. We cannot assign the correct counterpart with the available data, although the final SED resembles that of a young object.

SBO3 and SBO4 appear to be associated with B30-LB22, although SB04 is closer ( $\sim \operatorname{arcsec})$ to the LABOCA peak than SB03 ( 11"). In the case of SB03, the only possible detected counterpart is LB22f, while we can assign LB22a to SB04 (following the nomenclature by BGH16). LB22f is classified as a non-member by BGH16 and its SED does not, in fact, display any infrared excess. Therefore, we can assume that the infrared source is unrelated to SB03, which could be classified as a starless core. LB22a is classified as a B30 member with a peculiar SED (with only four points, see Fig. 9). The target is detected in CAHA/O2000 and IRAC/3.6 $\mu \mathrm{m}$ at a separation of 2 to $3 \operatorname{arcsec}$ from the SABOCA source. Finally, we note that the $8 \mu \mathrm{m}$ IRAC image shows extended emission close to SB04, with a tentative infrared detection at 4.7 arcsec (source \#b? in Fig. 9).

The two SABOCA sources SB05 and SB06 are close $\left(5.8^{\prime \prime}\right.$ and $8.0^{\prime \prime}$, respectively) to the peak of the LABOCA core B30LB23. SB05 is at a separation of 3.8 arcsec from the infrared

\footnotetext{
4 http://wise2.ipac.caltech.edu/docs/release/allwise/
} 

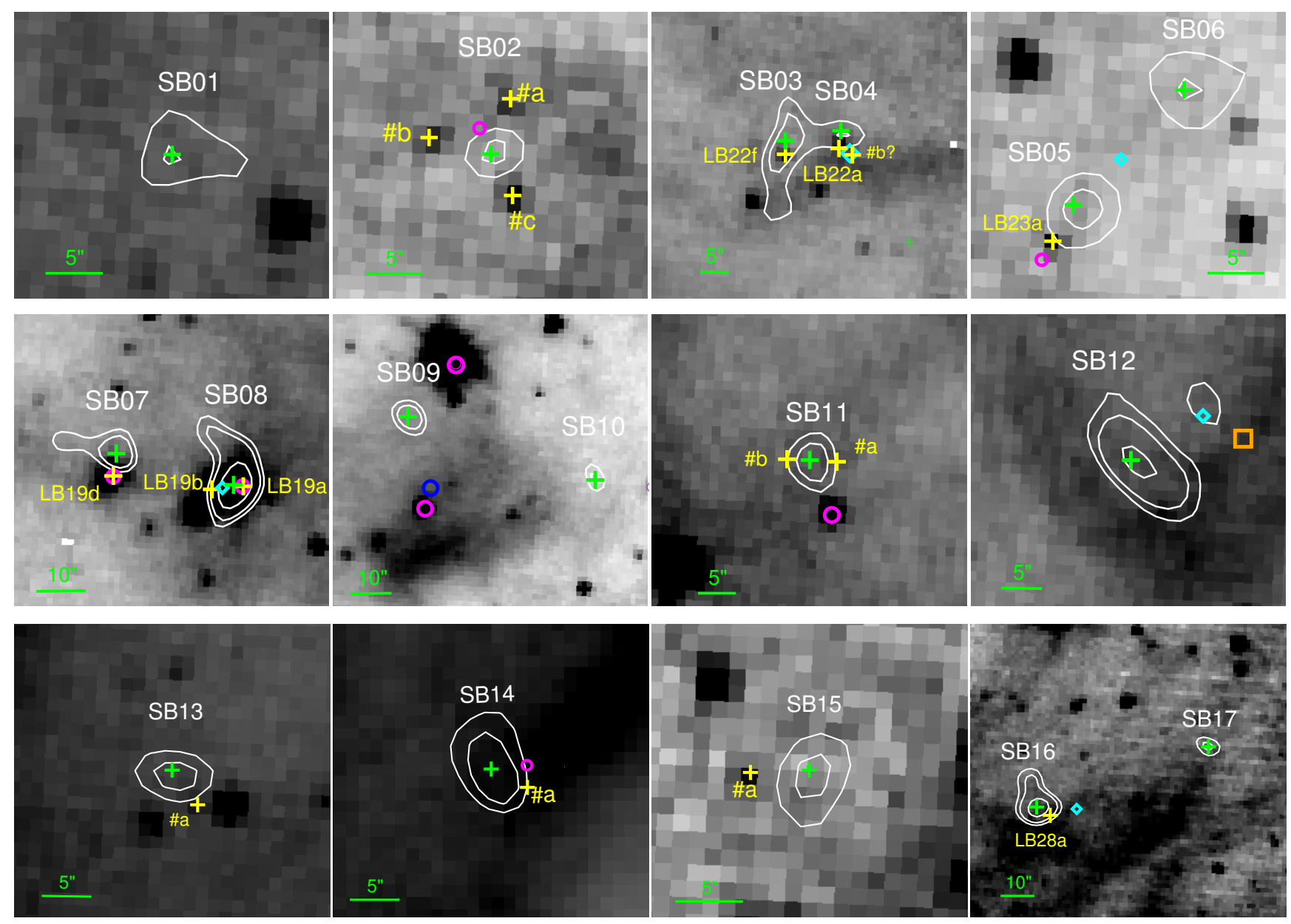

Fig. 8. Spitzer IRAC $3.6 \mu \mathrm{m}$ finding charts with the APEX/SABOCA detections, except for SB14 (IRAC $8 \mu \mathrm{m}$ image). North is up and east to the left. The green crosses represent the coordinates of the peak emission of the APEX/SABOCA detections. The white contours represent the APEX/SABOCA emission at 2.5, 3, 4 and $5 \sigma$ level. The infrared counterparts within a radius of 5":3 (half of the APEX/SABOCA beam) are represented by yellow crosses. If previously discussed by BGH16, they are named with the APEX/LABOCA designation. If not, they are named with low-case letters. The magenta circles represent WISE detections, the cyan diamonds represent the position of the APEX/LABOCA cores peak intensity, and the blue circles indicate $A K A R I$ detections. The orange square represents a Planck detection (see text).

source LB23a, which is classified as a probable member by BGH16. Its SED shows a clear IR excess starting at $\sim 6 \mu \mathrm{m}$. We also detect a source with WISE slightly further away (5.6" from the SABOCA peak coordinates, WISE J053129.66+120429.8) but, given the WISE angular resolution $\left(6.1^{\prime \prime}\right.$ at $\left.3.4 \mu \mathrm{m}\right)$, is probably related with LB23a. However, we think that if the infrared source LB23a (which is very compact) were related to the SABOCA and LABOCA emission, it should have been detected with ALMA when pointing at LB23, but it was not. Therefore, we can conclude that they are probably unrelated. Since the ALMA LAS is 6", the SABOCA and LABOCA emission have to be associated with a structure larger than $6^{\prime \prime}$ (or $2400 \mathrm{AU}$ at $400 \mathrm{pc}$ ). In fact, it is also possible that the Spitzer/MIPS $70 \mu \mathrm{m}$ emission is also unrelated to the compact infrared source but associated to SB05 and LB23. The source SB06 does not show any infrared counterpart, and can be considered a starless core.

For SB07 we detect an IR counterpart at a separation of 4.9 arcsec. This is a very bright source close to B30-LB19, and named LB19d by BGH16. It is classified as a member of B30, and its SED shows a clear infrared excess. Curiously, this object is included in the NED database and classified as a galaxy ("G"), based on its inclusion in the 2MASS Catalogue of extended sources. To the best of our knowledge there is no other evidence pointing towards an extragalactic nature according to the NED database. As seen in Fig. 10, the object is resolved into two sources in our CAHA/O2000 near-IR infrared images, and displays some diffuse extended emission that resembles that of a bipolar nebula. The infrared counterpart LB19d is also detected with WISE (J053129.61+120532.8) and the final SED is typical of a young stellar object.

As already discussed in Sect. 3.1.2. SB08 is LB19. The infrared counterpart, LB19a, is detected at a separation of 1.7".

The source SB09 does not have any counterpart in the nearIR, Spitzer or WISE data. There is a detection with AKARI/FIS at a separation of 19" SW (namely, 0531219+120513, see SB09 panel in Fig. 8), however this AKARI source is located at $\sim 5^{\prime \prime}$ from the bright WISE source WISE J053121.99+120507.9 (LB25c in BGH16), so they are probably associated. Therefore, SB09 remains as a probable starless core.

For SB11 we detected two possible counterparts in the Spitzer/IRAC images. One of them is detected at 3.5 and $4.5 \mu \mathrm{m}$ at a separation of 3.6". The second one is very faint and only detected at the IRAC I4 channel at $8.0 \mu \mathrm{m}$ at a separation of $3.1^{\prime \prime}$ With this information it is difficult to assign the correct counterpart. There is one bright mid-IR source detected (with WISE too), but at $7.5^{\prime \prime}$ from the source. 

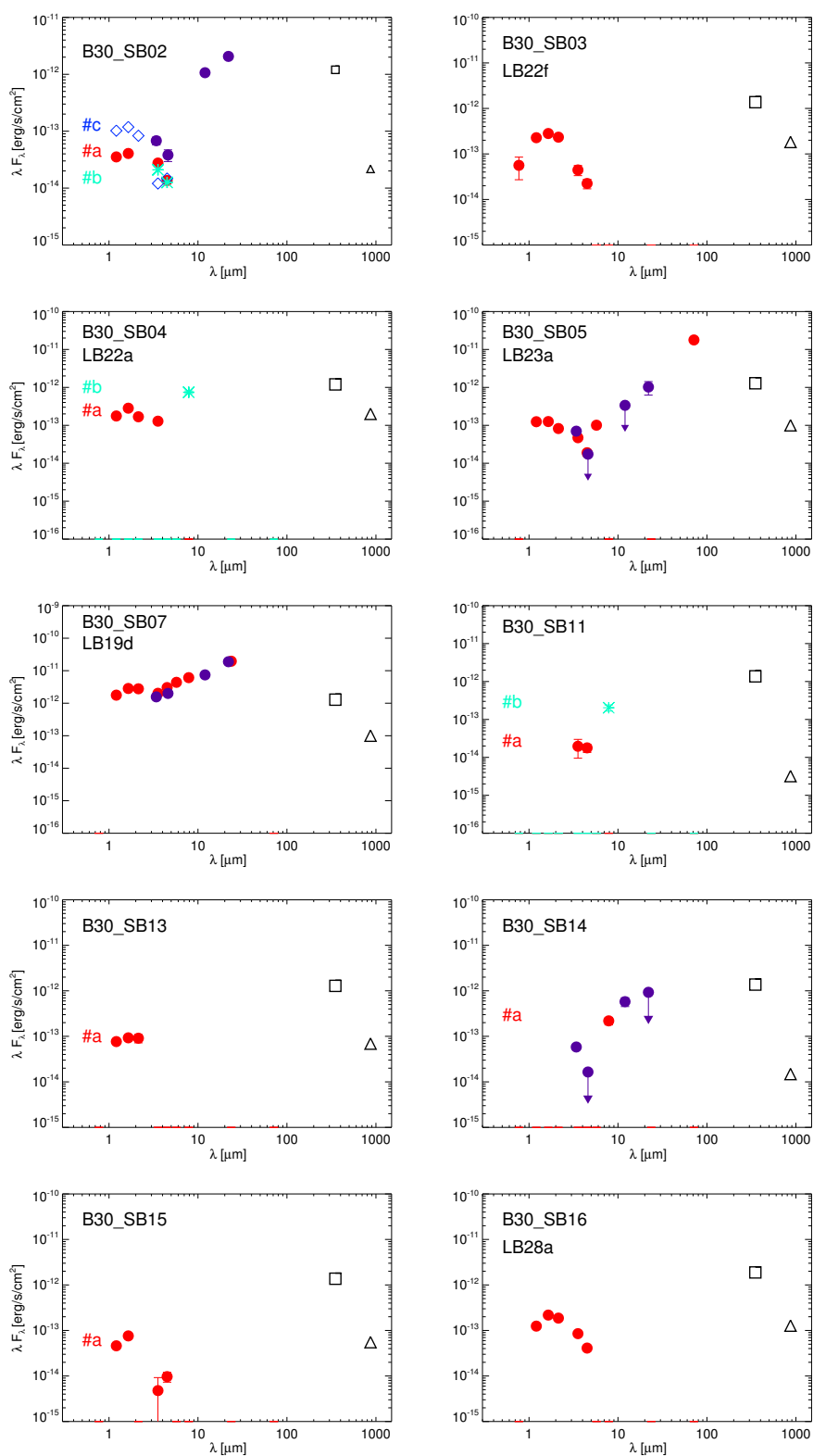

Fig. 9. SEDs of the infrared counterparts to the APEX/SABOCA detections. Those with multiple counterparts are plotted with different colors. The purple circles represent WISE data available for some of the sources.

The SB12 source does not have any counterpart in the nearIR, Spitzer or WISE data. There is, however, a detection in the Planck Catalog of compact sources, G192.65-11.70, at a separation of 14.2" from SB12, and at 5.5" from the APEX/LABOCA source B30-LB27, classified as an extended source. The fact that the latter is not detected with ALMA points towards an extended clump (the angular size measured with APEX/LABOCA is 29") and its nature (transient or not) is unclear, but see BGH16.

For SB13 there is only one counterpart detected at $4.4^{\prime \prime}$ in the near-IR. The source is marginally seen at $3.6 \mu \mathrm{m}$. The fact that is not detected at longer IRAC wavelengths suggests that is probably a contaminant.

Only one counterpart is detected for SB14 in the $8 \mu \mathrm{m}$ IRAC image at $4.2^{\prime \prime}$ separation. There is a source detected with WISE that seems to be related to the IRAC detection, although with SNR between 3 and 10 at $3.4 \mu \mathrm{m}$ and $12 \mu \mathrm{m}$, and upper limits

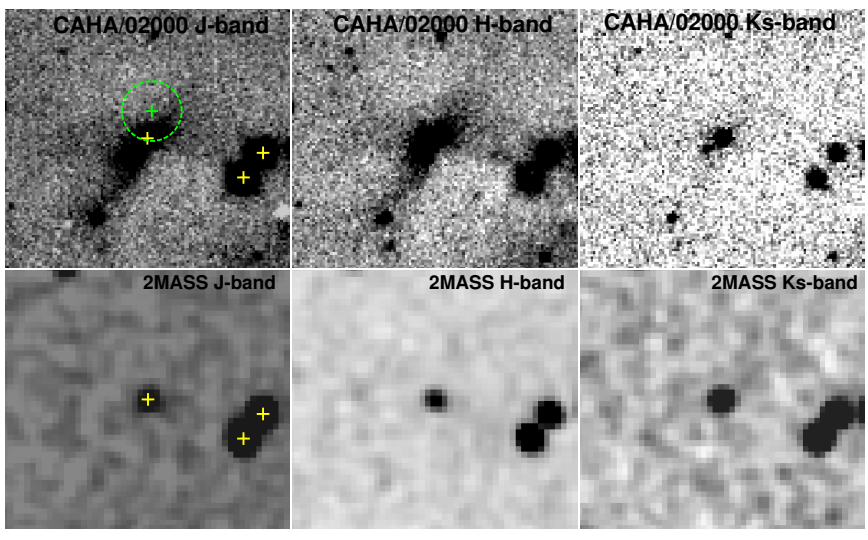

Fig. 10. Infrared images of the source SB07 (LB19d). North is up and east to the left. The upper panels show our CAHA/O2000 JHK $\mathrm{im}$ ages, and the lower ones the 2MASS images. We spatially resolve the object into two sources. The $J$ and $H$-band images show diffuse extended emission resembling a bipolar nebula. In the upper left panel we have represented the peak intensity coordinates of the SABOCA source SB07 (green cross), the SABOCA beam size (10.6" diameter) and the 2MASS detections in the field (yellow crosses in the $J$-band images).

in the other two bands (WISE J053111.14+120624.4). If the infrared source is related with SB12, the SED is consistent with a young object, although additional data is needed for its characterization.

For SB15 there is an IR counterpart located $5^{\prime \prime}$ from the SABOCA detection. The SED only includes four data points with a very uncertain $3.5 \mu \mathrm{m}$ measurement. We cannot conclude if it is related to the SABOCA source.

Only one infrared counterpart was found for SB16 at 4.6" (LB28a in BGH16). It is classified as a member, but its SED (with points up to $4.5 \mu \mathrm{m}$ ) does not show a clear infrared excess, therefore the IR and sub-mm sources are probably unrelated.

Finally, we note that, in addition to SB06, we did not find IR counterparts to the SABOCA sources SB01, SB09 (B30-LB20), SB10, SB12 (B30-LB27), and SB17. In the case of SB06, SB09 and SB12, they are associated with LABOCA cores, however we verified that they were not included in the field of view of the ALMA images. This is not the case for SB03, which is included in one of the ALMA pointings, but is undetected. Therefore, we remain with six SABOCA sources that are good candidates to be starless cores. Higher quality observations will help us to understand the true nature of these sources, since our SABOCA map is not sensitive enough to make a detailed study.

\section{Discussion}

The ALMA data have allowed us to characterize two very young stellar members of B30 through the analysis of their continuum and gas emission. We have also detected three sources without IR counterparts.

In the following subsections, we discuss the low rate of ALMA detections in the sample of observed APEX/LABOCA cores, and compare the properties of the cores with and without ALMA detections. Finally, we also discuss the possible nature of the three ALMA detections without IR counterparts.

\subsection{ALMA detection rate}

We observed a total of 30 cores previously detected with APEX/LABOCA and with flux densities between $\sim 30$ and 
$\sim 190 \mathrm{mJy}$, with $S N R \geq 3$, and mainly spatially unresolved (see Table 2 from BGH16). Since the APEX/LABOCA beam is $\sim 27^{\prime \prime}$, we estimate sizes smaller than $\sim 10800 \mathrm{AU}$ in diameter for the unresolved cores.

In the ALMA observations presented here we have only detected 5 out of 30 sources, corresponding to a detection rate of $17 \%$. This can be related to our sensitivity or/and to the size of the emitting sources. The average rms of our ALMA observations is $\sim 0.13 \mathrm{mJy}$. In the case of the faintest APEX/LABOCA source, with a flux density of $\sim 29 \mathrm{mJy}$, we would expect a flux per beam of $0.8 \mathrm{mJy}$ if we assume the less favourable case in which the size of the source is as large as the size given by the ALMA LAS $\left(\sim 6^{\prime \prime}\right)$. Even in this unfavourable case, ALMA could have detected the APEX/LABOCA sources with $S N R \geq 6$. Since we do not detect most of the APEX/LABOCA cores, we conclude that the low rate of ALMA detections is related to the size of the emitting sources. Based on the LAS, we estimate that most of the APEX/LABOCA cores have sizes larger than $2400 \mathrm{AU}$ (6" at $400 \mathrm{pc}$ ), or $1200 \mathrm{AU}$ in radius, as also suggested by the fact that in all but one of the ALMA detections (LB14) the flux missed by ALMA is extremely large ( $>85 \%$, see Table 1$)$. These APEX/LABOCA cores, which remained undetected by ALMA and seem to be dominated by large-scale emission, may be transient cores.

\subsection{Comparison of LABOCA cores with and without ALMA detections}

We compared the properties of the LABOCA cores with and without ALMA detections. First of all, we compared the spatial distribution of the five ALMA sources within the B30 cloud. They are located both in the northern parts of the cloud (LB08, LB10 and LB14) and in the ionization front (LB19 and LB31). This is also the case for the 25 LABOCA sources undetected with ALMA (see Fig. 1), so we do not see any relation between the detected sources and their position within the cloud.

Regarding the masses, the five ALMA detections are associated with APEX/LABOCA cores with masses between 0.046 and $0.182 M_{\odot}$, comparable with the undetected cores with masses between 0.043 and $0.278 M_{\odot}$.

Finally, we compared the estimated sizes: from the 30 cores observed with ALMA, only eight were spatially resolved with APEX/LABOCA, with sizes (diameters) between 27" and 62" (this is the deconvolved size after fitting a Gaussian to the cores, see BGH16). In the case of ALMA, we detected five point-like sources, only one being spatially resolved in the LABOCA data: LB08 with 27".

Therefore, we do not report any particular property of the ALMA detected cores when compared with the non-detected ones. As explained in the previous subsection, the main difference should be that the APEX/LABOCA cores undetected with ALMA have most of their mass in large scales (scales larger than the LAS of ALMA observations).

\subsection{Nature of ALMA detections without infrared counterparts}

If we assume that LB08, LB10 and LB31 are associated with B30, and since they do not show IR counterparts up to $24 \mu \mathrm{m}$ (in the case of LB08, up to $70 \mu \mathrm{m}$ ), they could be classified either as deeply embedded protostars undetected in the Spitzer observations (see e.g., Stutz et al. 2013), or as starless cores.

In the protostar scenario, we can provide a limit to the luminosity for the central sources. LB08 is undetected in the MIPS
$70 \mu \mathrm{m}$ image, however we have estimated a background intensity at the ALMA position of $\sim 0.22 \mathrm{Jy} /$ beam. If LB08 had a deeply embedded and undetected counterpart at this wavelength, we could use this flux as an upper limit to the emission of a source at the given position. We could then estimate an upper limit to the internal luminosity of a potential counterpart using the formula by Dunham et al. (2008), scaled to the distance of B30. By doing this, we derived a value of $L_{\text {int }}<0.1 L_{\odot}$, which is the luminosity limit established for VeLLOs. As a different approach, we can use the limiting magnitudes at all wavelengths (they have been estimated in BGH16, see their Table 1), together with the APEX/LABOCA fluxes (instead of the ALMA fluxes that can suffer significant levels of spatial filtering when compared with single-dish observations), to integrate the SED of the object (see Fig. 5) and derive upper limits to the bolometric luminosity and bolometric temperature. We estimate $L_{\text {bol }}<0.08 L_{\odot}$ and $T_{\text {bol }}>54 \mathrm{~K}$, respectively. These values suggest that the source could be a VeLLO, with a lower limit of the $T_{\text {bol }}$ consistent with a Class 0 object (Class 0 sources show $T_{\text {bol }}<90 \mathrm{~K}$, Chen et al. 1995) or more evolved. The value of $L_{\text {bol }}<0.07 L_{\odot}$ implies that the internal luminosity should be even smaller.

In the case of LB10 and LB31, there are no $70 \mu \mathrm{m}$ data available at their positions. Since LB31 has an upper limit at $350 \mu \mathrm{m}$ we can estimate $L_{\text {bol }}<0.05 L_{\odot}$ and $T_{\text {bol }}>21 \mathrm{~K}$ by integrating its SED (displayed in Fig. 5). This means that LB31 could also be a VeLLO with a $T_{\text {bol }}$ consistent with a Class 0 (or more evolved) object, although this is a very rough estimation due to the lack of data-points in the sub-mm range. The fact that this source is well within the ionization front of the B30 cloud (see Fig. 1) suggests that it could be a photo-evaporated proto-BD. In the case of LB10, there is no data available between $24 \mu \mathrm{m}$ and $870 \mu \mathrm{m}$. Hence, it is difficult to estimate a limit for the luminosity for this object, since the sub-mm range is not well-sampled.

A second possibility that we explored is that the three ALMA sources are starless cores. For a $T_{\mathrm{d}}$ of $15 \mathrm{~K}$, their estimated masses are between 0.9 and $9 M_{\text {Jup }}$ using the ALMA data, and between $\sim 46$ and $106 M_{\text {Jup }}$ using the APEX/LABOCA data. Of special interest are the cases of LB10 and LB31, with APEX/LABOCA masses below $\left(46 M_{\text {Jup }}\right)$ or very close to the substellar regime $\left(82 M_{\mathrm{Jup}}\right)$, implying that they could be presubstellar cores.

If these three cores collapse to form stars, we can estimate the final masses of the central objects assuming that the core formation efficiency in low-mass dense cores is $\sim 30 \%$ (e.g., Motte et al. 1998; Alves et al. 2007; Bontemps et al. 2010). If we add $30 \%$ of the LABOCA mass to the ALMA masses, we obtain $\sim 41 M_{\text {Jup }}(\mathrm{LB} 08), \sim 17 M_{\text {Jup }}$ (LB10), and $\sim 26 M_{\text {Jup }}(\mathrm{LB} 31)$, all of them below the sub-stellar mass regime. Therefore, the three ALMA sources could be considered as pre-BD core candidates. We note that the derived masses are strongly dependent on the dust temperature. A colder temperature, such as $10 \mathrm{~K}$, would increase the final masses by a factor of approximately two, which would also be consistent with pre-BD core candidates in LB10 and LB31. LB08 would be at the border between a low-mass prestellar object and a pre-BD core.

In the framework of the turbulent fragmentation theory, Padoan \& Nordlund (2004) have explained that substellar objects can form in cores with a density as high as the critical density for the collapse of a BD mass core. To study if the three LABOCA starless cores detected with ALMA might form BDs, we followed the same procedure as de Gregorio-Monsalvo et al. (2016), and compared the density of the LABOCA cores with the critical density $\left(n_{\text {crit }}\right)$ of a Bonnor-Ebert $(\mathrm{BE})$ isothermal sphere, 
N. Huélamo et al.: A search for pre- and proto-brown dwarfs in the dark cloud Barnard 30 with ALMA

Table 3. Estimated critical densities (at $15 \mathrm{~K}$ ) and maximum radii for the three ALMA pre-BD core candidates to be gravitationally unstable.

\begin{tabular}{|c|c|c|c|c|c|c|c|c|c|c|}
\hline \multirow[b]{2}{*}{ Core } & \multicolumn{5}{|c|}{ APEX/LABOCA } & \multicolumn{5}{|c|}{ ALMA } \\
\hline & $\begin{array}{l}n_{\mathrm{crit}^{a}}{ }^{a} \\
{\left[\mathrm{~cm}^{-3}\right]}\end{array}$ & $\begin{array}{l}n_{\mathrm{obs}}{ }^{b} \\
{\left[\mathrm{~cm}^{-3}\right]}\end{array}$ & $\begin{array}{l}R_{\max }{ }^{c} \\
{[\mathrm{AU}]}\end{array}$ & $\begin{array}{l}R_{\mathrm{obs}}{ }^{d} \\
{[\mathrm{AU}]}\end{array}$ & $\begin{array}{l}\text { Dynamical } \\
\text { state }\end{array}$ & $\begin{array}{l}n_{\mathrm{crit}^{a}}{ }^{a} \\
{\left[\mathrm{~cm}^{-3}\right]}\end{array}$ & $\begin{array}{l}n_{\mathrm{obs}}{ }^{b} \\
{\left[\mathrm{~cm}^{-3}\right]}\end{array}$ & $\begin{array}{l}R_{\max }{ }^{c} \\
{[\mathrm{AU}]}\end{array}$ & $\begin{array}{l}R_{\mathrm{obs}}{ }^{d} \\
{[\mathrm{AU}]}\end{array}$ & $\begin{array}{l}\text { Dynamical } \\
\text { state }\end{array}$ \\
\hline LB08 & $3.3 \times 10^{6}$ & $2.5 \times 10^{4}$ & 971 & 5400 & stable/transient & $5.0 \times 10^{8}$ & $>1.3 \times 10^{8}$ & 82 & $<140$ & infalling? \\
\hline LB 10 & $1.7 \times 10^{7}$ & $>1.0 \times 10^{4}$ & 421 & $1200^{*}-5520$ & stable? & $3.4 \times 10^{9}$ & $>4.9 \times 10^{7}$ & 28 & $<140$ & infalling? \\
\hline LB31 & $5.5 \times 10^{6}$ & $>1.8 \times 10^{4}$ & 751 & $1200^{*}-5520$ & stable? & $4.5 \times 10^{10}$ & $>1.3 \times 10^{7}$ & 8 & $<140$ & infalling? \\
\hline
\end{tabular}

Notes. ${ }^{(a)}$ Critical density estimated following Eq. (3), and using the corresponding APEX/LABOCA or ALMA mass for a temperature of 15 K, as given in Table 1; ${ }^{(b)}$ Measured density using the APEX/LABOCA or ALMA masses given in Table 1, obtained for a temperature of $15 \mathrm{~K}$, and using the measured size or the beam size as upper limit if the source is unresolved; ${ }^{(c)}$ Maximum radii corresponding to the critical density given in this table, and using the mass used to obtain the critical density; ${ }^{(d)}$ Measured radii in the APEX/LABOCA or ALMA observations. If the sources are unresolved we give half of the beam size as un upper limit for the radius. The lower limit of the radius in the APEX/LABOCA column corresponds to half the LAS. We adopted this value as a lower limit for the radius of the APEX/LABOCA core given that $>90 \%$ of the flux is filtered out by ALMA.

following the relation:

$\left[\frac{n_{\text {crit }}}{\mathrm{cm}^{-3}}\right]=1.089 \times 10^{4}\left[\frac{M_{\mathrm{BE}}}{M_{\odot}}\right]^{-2}\left[\frac{T}{10 \mathrm{~K}}\right]^{3}$,

where $M_{\mathrm{BE}}$ is the mass of the $\mathrm{BE}$ sphere. For these three cores, we have first assumed that the mass of the BE sphere is the one obtained with LABOCA for a typical temperature of $15 \mathrm{~K}$ (Table 1), and calculated $n_{\text {crit }}$ following Eq. (3). We also estimated, given the mass of the object, the radius $R_{\max }$ corresponding to $n_{\text {crit }}$, and compared it to the measured radius (only LB08 is resolved, for the other cores we took the beam size as an upper limit). The results are shown in Table 3.

Table 3 shows that, if we take into account only APEX/LABOCA data, LB08 is not gravitationally unstable, because its density is smaller than the critical density by approximately two orders of magnitude, and its radius is larger than the maximum radius required for instability. For the case of LB10 and LB31, this is not so clear, because they are not resolved by APEX and the observed densities are only lower limits. However, if we compare $R_{\max }$ with the possible sizes of the cores, we again find that also LB10 and LB31 should not be gravitationally unstable. This is because, although the cores are not resolved, ALMA has filtered out even more flux than for the case of LB08 (i.e., >90\%, see Table 1), strongly suggesting that most of the mass measured by APEX/LABOCA comes from spatial scales much larger than the ALMA LAS, that is, much larger than $1200 \mathrm{AU}$, and probably larger than $R_{\max }$, especially for the case of LB10. Thus, using only the APEX/LABOCA data, it would rather seem, following the Padoan \& Nordlund (2004) theory, that the three cores are not gravitationally unstable.

This poses a major problem, because ALMA has revealed that there already seems to be a very compact object at the center of the APEX/LABOCA cores, indicative of on-going collapse. In fact, the calculated upper limits to the observed densities using ALMA remain consistent with infalling objects (mainly in the case of LB08, which is of the same order as the estimated critical density). In addition, very recent studies conclude that a shock condition for the turbulence fragmentation scenario to be efficient is a very unlikely configuration of converging flows (Lomax et al. 2016), again posing serious problems to the turbulent fragmentation theory for BD formation.

An alternative scenario is to assume that the three ALMA pre-BD core candidates are already the result of gravitational contraction, constituting the "tip of the iceberg" of a larger-scale collapse. In this case, the collapse
Table 4. Estimated masses (at $15 \mathrm{~K}$ ) of the three ALMA pre-BD core candidates within an ALMA beam assuming a $r^{-2}$ density profile.

\begin{tabular}{lllll}
\hline \hline & \multicolumn{2}{l}{ APEX/LABOCA } & \multicolumn{2}{c}{ ALMA Mass } \\
Mass & Radius & Estimated* & Observed \\
Core & {$\left[M_{\text {Jup }}\right]$} & {$[\mathrm{AU}]$} & {$\left[M_{\text {Jup }}\right]$} & {$\left[M_{\text {Jup }}\right]$} \\
\hline LB08 & 106 & 5400 & 3 & 9 \\
LB10 & 46 & 5520 & 1 & 3 \\
LB31 & 82 & 5520 & 2 & 0.9 \\
\hline
\end{tabular}

Notes. ${ }^{(*)}$ Estimated for an average beam size of $0.7^{\prime \prime}$, or $140 \mathrm{AU}$ of radius.

is expected to occur from the outside-in and to selfconsistently develop a near $r^{-2}$ density profile but with a finite infall velocity during their prestellar evolution (e.g., Larson 1969; Whitworth \& Summers 1985; Gómez et al. 2007; Gong \& Ostriker 2009; Mohammadpour \& Stahler 2013; Naranjo-Romero et al. 2015). If the cores have indeed an $r^{-2}$ profile, and we assume that the masses inside the APEX beam are the ones given in Table 1, we can estimate the expected masses inside the ALMA beam (since we measure $\sim 0.9^{\prime \prime} \times 0.5^{\prime \prime}$, we have assumed an average value of 0.7 arcsec, corresponding to $140 \mathrm{AU}$ of radius). The result is displayed in Table 4 . The estimated masses are $\sim 3,1$, and $2 M_{\text {Jup }}$ for LB08, LB10 and LB31, respectively. Interestingly, they are consistent with the measured ones within the uncertainties (typically of a factor of 4), suggesting that the density profile is indeed not far from $r^{-2}$, and supporting the hypothesis that the whole core is already the result of a gravitational collapse. Further observations aimed at studying the kinematics of the three ALMA sources would help to elucidate whether or not their hosting cores are indeed infalling towards the center. These observations would also help to estimate the final mass of the future collapsed objects and thus assess (or not) their pre-BD nature.

Finally, we stress that with our current data set we cannot exclude the possibility that these three sources are extragalactic contaminants not included in the NED database. Although their location in the cloud (well within the filaments, at least in the case of LB08 and LB31) suggests that they are probably associated to B30, future ALMA observations will allow confirmation of their B30 membership. 


\section{Conclusions}

We analyzed ALMA observations of the young dark cloud B30 at $880 \mu \mathrm{m}$. We complemented the ALMA observations with APEX/SABOCA data obtained and $350 \mu \mathrm{m}$ and covering the southern part of the B30 cloud. The aim of our study was to shed light on the nature of 30 sub-mm sources previously detected with APEX/LABOCA at $870 \mu \mathrm{m}$. Our main results can be summarized as follows:

- We have detected 5 (out of 30) compact sources with ALMA with masses between 0.9 and $67 M_{\text {Jup }}$. Two of them show clear infrared counterparts (LB14 and LB19), while three of them do not (LB08, LB10 and LB31).

- The properties of LB14 and LB19 are consistent with a Class II and a Class I low-mass stellar object, respectively. Both sources show gas emission that allows for their confirmation as B30 members. LB14 shows very compact gas emission compatible with a rotating disk. LB19 shows two gas emission components: a compact one, with an asymmetric spectrum in which the blueshifted emission is stronger than the red one, and an extended component of $\sim 2400 \mathrm{AU}$ surrounding the compact emission. The kinematical analysis of the two components suggests that we might be observing a rotating oblate envelope, and infalling signatures at $400 \mathrm{AU}$ scales.

- If LB08, LB10 and LB31 belong to B30, they can be classified as either deeply embedded protostars, or starless cores. In the former scenario, two of the sources (LB08 and LB31) show internal luminosity upper limits consistent with VeLLOs, while LB10 does not have enough data to estimate a reliable limit. In the latter scenario, the estimated final masses of the three sources are near the substellar regime. Therefore, they could be pre-BD core candidates if B30 members. One of the sources (LB31) is located at the ionization front of the B30 cloud and might be a photo-evaporated proto-BD or pre-BD core candidate.

- According to the theory of BD formation through turbulent fragmentation, the three starless LABOCA cores with ALMA detections are more consistent with gravitationally stable cores. However, this result is not consistent with the fact that ALMA detected a compact source at the center of these cores. As an alternate scenario, we propose that these cores are the result of a large scale gravitational contraction. In this case, the estimated masses using a $r^{-2}$ density profile (which is characteristic of a collapsing core) are consistent with the observed ones within the uncertainties.

- We detected 17 sources with SABOCA at $350 \mu \mathrm{m}$ in the southern part of the cloud but with very low SNR (between 3.0 and 4.6). Eleven show infrared counterparts, although in most of the cases they are detected at separation larger than $4^{\prime \prime}$ from the center of the SABOCA beam, so their association is uncertain. Six objects do not show IR counterparts and may be considered starless cores.

Future observations will allow confirmation of the true nature of the three ALMA sources without IR counterparts.

Acknowledgements. This project has been funded by the BBVA Foundation under the Convocatoria 2015 de Ayudas Fundación BBVA a Investigadores y Creadores Culturales. We thank E. Vázquez-Semadeni and J. Alves for very useful comments, and R. Lorente and I.Yamamura for their help with the AKARI data. We are indebted to the Calar Alto Observatory staff for their excellent work taking the near-IR data under the Service Mode program. NH thanks the ALMA Science Center in Santiago for hosting her during 2.5 months. I.d.G. acknowledges support fromMICINN (Spain) AYA2011-30228-C03 grant (including
FEDER funds). A.P. and L.Z. acknowledge the financial support from UNAMDGAPA-PAPITT IA102815 grant (México). D.B. has been funded by Spanish grant AYA2012-38897-C02-01. H.B. is funded by the Ramón y Cajal fellowship program number RYC-2009-04497. A.B. acknowledges financial support from the Proyecto Fondecyt de Iniciación 11140572, and scientific support from the Millenium Science Initiative, Chilean Ministry of Economy, Nucleus RC130007. M.T.R. acknowledges partial support from CATA (PB06, CONICYT-Chile). C.E. is partly supported by Spanish Grant AYA 2014-55840-P. This publication makes use of data products from the Wide-field Infrared Survey Explorer, which is a joint project of the University of California, Los Angeles, and the Jet Propulsion Laboratory/California Institute of Technology, funded by the National Aeronautics and Space Administration.

\section{References}

Allard, F., Homeier, D., Freytag, B., \& Sharp, C. M. 2012, in EAS PS 57, eds. C. Reylé, C. Charbonnel, \& M. Schultheis, 3

Allen, L. E., Calvet, N., D’Alessio, P., et al. 2004, ApJS, 154, 363

Alves, J., Lombardi, M., \& Lada, C. J. 2007, A\&A, 462, L17

Alves de Oliveira, C., Moraux, E., Bouvier, J., \& Bouy, H. 2012, A\&A, 539, A151

André, P., Ward-Thompson, D., \& Greaves, J. 2012, Science, 337, 69

Barrado y Navascués, D., \& Jayawardhana, R. 2004, ApJ, 615, 840

Barrado, D., Morales-Calderón, M., Palau, A., et al. 2009, A\&A, 508, 859

Barrado, D., de Gregorio-Monsalvo, I., Huelamo, N., et al. 2016, A\&A, submitted

Barrado y Navascués, D., Stauffer, J. R., Bouvier, J., Jayawardhana, R., \& Cuillandre, J.-C. 2004, ApJ, 610, 1064

Barrado y Navascués, D., Stauffer, J. R., Morales-Calderón, M., et al. 2007, ApJ, 664,481

Bate, M. R. 2012, MNRAS, 419, 3115

Bate, M. R., Bonnell, I. A., \& Bromm, V. 2002, MNRAS, 332, L65

Bayo, A. 2009, Ph.D. dissertation, Universidad Autónoma de Madrid, Spain

Bayo, A., Barrado, D., Stauffer, J., et al. 2011, A\&A, 536, A63

Bonnell, I. A., Clark, P., \& Bate, M. R. 2008, MNRAS, 389, 1556

Bontemps, S., Motte, F., Csengeri, T., \& Schneider, N. 2010, A\&A, 524, A18

Bourke, T. L., Myers, P. C., Evans, II, N. J., et al. 2006, ApJ, 649, L37

Bouy, H., Huélamo, N., Martín, E. L., et al. 2007, A\&A, 463, 641

Bouy, H., Huélamo, N., Martín, E. L., et al. 2009, A\&A, 493, 931

Caballero, J. A., Béjar, V. J. S., Rebolo, R., et al. 2007, A\&A, 470, 903

Cambresy, L., Epchtein, N., Copet, E., et al. 1997, A\&A, 324, L5

Chen, H., Myers, P. C., Ladd, E. F., \& Wood, D. O. S. 1995, ApJ, 445, 377

Connelley, M. S., Reipurth, B., \& Tokunaga, A. T. 2008, AJ, 135, 2496

de Gregorio-Monsalvo, I., Barrado, D., Bouy, H., et al. 2016, A\&A, 590, A79

Delfosse, X., Tinney, C. G., Forveille, T., et al. 1997, A\&A, 327, L25

Delorme, P., Willott, C. J., Forveille, T., et al. 2008, A\&A, 484, 469

di Francesco, J., Evans, II, N. J., Caselli, P., et al. 2007, Protostars and Planets V, 17

Dolan, C. J., \& Mathieu, R. D. 1999, AJ, 118, 2409

Dolan, C. J., \& Mathieu, R. D. 2001, AJ, 121, 2124

Dolan, C. J., \& Mathieu, R. D. 2002, AJ, 123, 387

Duerr, R., Imhoff, C. L., \& Lada, C. J. 1982, ApJ, 261, 135

Dunham, M. M., Crapsi, A., Evans, II, N. J., et al. 2008, ApJS, 179, 249

Fitzpatrick, E. L. 1999, PASP, 111, 63

Forbrich, J., Lada, C. J., Lombardi, M., Román-Zúñiga, C., \& Alves, J. 2015, A\&A, 580, A114

Gómez, G. C., Vázquez-Semadeni, E., Shadmehri, M., \& Ballesteros-Paredes, J. 2007, ApJ, 669, 1042

Gomez, M., \& Lada, C. J. 1998, AJ, 116, 1508

Gong, H., \& Ostriker, E. C. 2009, ApJ, 699, 230

Hennebelle, P., \& Chabrier, G. 2008, ApJ, 684, 395

Hester, J. J., Scowen, P. A., Sankrit, R., et al. 1996, AJ, 111, 2349

Hodapp, K. W., Iserlohe, C., Stecklum, B., \& Krabbe, A. 2009, ApJ, 701, L100

Kovács, A. 2008, in Millimeter and Submillimeter Detectors and Instrumentation for Astronomy IV, Proc. SPIE, 7020, 70201S

Lang, W. J., Masheder, M. R. W., Dame, T. M., \& Thaddeus, P. 2000, A\&A, 357, 1001

Larson, R. B. 1969, MNRAS, 145, 271

Lee, C. W., Bourke, T. L., Myers, P. C., et al. 2009, ApJ, 693, 1290

Lee, C. W., Kim, M.-R., Kim, G., et al. 2013, ApJ, 777, 50

Liu, T., Zhang, Q., Kim, K.-T., et al. 2016, ApJS, 222, 7

Lomax, O., Whitworth, A. P., \& Hubber, D. A. 2016, MNRAS, 458, 1242

Mainzer, A., Cushing, M. C., Skrutskie, M., et al. 2011, ApJ, 726, 30

Matzner, C. D., \& Levin, Y. 2005, ApJ, 628, 817

Mohammadpour, M., \& Stahler, S. W. 2013, MNRAS, 433, 3389 
Morales-Calderón, M. 2008, Ph.D. dissertation, Universidad Autónoma de Madrid, Spain

Morata, O., Palau, A., González, R. F., et al. 2015, ApJ, 807, 55

Motte, F., Andre, P., \& Neri, R. 1998, A\&A, 336, 150

Murdin, P., \& Penston, M. V. 1977, MNRAS, 181, 657

Mužić, K., Scholz, A., Geers, V. C., \& Jayawardhana, R. 2015, ApJ, 810, 159

Naranjo-Romero, R., Vázquez-Semadeni, E., \& Loughnane, R. M. 2015, ApJ, 814,48

Ossenkopf, V., \& Henning, T. 1994, A\&A, 291, 943

Padoan, P., \& Nordlund, A. 2004, ApJ, 617, 559

Palau, A., de Gregorio-Monsalvo, I., Morata, Ò., et al. 2012, MNRAS, 424, 2778

Palau, A., Sánchez-Monge, Á., Busquet, G., et al. 2010, A\&A, 510, A5

Palau, A., Zapata, L. A., Rodríguez, L. F., et al. 2014, MNRAS, 444, 833

Perryman, M. A. C., Lindegren, L., Kovalevsky, J., et al. 1997, A\&A, 323, L49
Planck Collaboration XI. 2014, A\&A, 571, A11

Reid, M. J., Schneps, M. H., Moran, J. M., et al. 1988, ApJ, 330, 809

Reipurth, B., \& Clarke, C. 2001, AJ, 122, 432

Scholz, A., Geers, V., Clark, P., Jayawardhana, R., \& Muzic, K. 2013, ApJ, 775, 138

Stutz, A. M., Tobin, J. J., Stanke, T., et al. 2013, ApJ, 767, 36

White, R. J., \& Basri, G. 2003, ApJ, 582, 1109

Whitworth, A., \& Summers, D. 1985, MNRAS, 214, 1

Whitworth, A. P., \& Stamatellos, D. 2006, A\&A, 458, 817

Whitworth, A. P., \& Zinnecker, H. 2004, A\&A, 427, 299

Yamamura, I., Makiuti, S., Ikeda, N., et al. 2009, in AKARI, a Light to Illuminate the Misty Universe, eds. T. Onaka, G. J. White, T. Nakagawa, \& I. Yamamura, ASP Conf. Ser., 418, 3

Young, C. H., Jørgensen, J. K., Shirley, Y. L., et al. 2004, ApJS, 154, 396

\section{Appendix A: ALMA observations centered at the APEX/LABOCA core detection coordinates}

Table A.1. ALMA observation $\log$.

\begin{tabular}{|c|c|c|c|c|c|}
\hline ALMA pointing & LABOCA Designation $^{a}$ & $\begin{array}{l}\text { RA }(\mathrm{J} 2000)^{b} \\
{[\mathrm{~h} \mathrm{~m} \mathrm{~s}]}\end{array}$ & $\begin{array}{l}\operatorname{Dec}^{b} \\
{\left[{ }^{\circ},{ }^{\prime},\right]}\end{array}$ & $\begin{array}{l}\text { Continuum rms } \\
{[\mathrm{mJy} / \text { beam }]}\end{array}$ & Detection? \\
\hline 1 & LB30 & $05: 31: 13.4$ & $+12: 03: 34.7$ & 0.13 & No \\
\hline 2 & LB31 & $05: 31: 15.3$ & $+12: 03: 38.7$ & 0.13 & YES \\
\hline 3 & LB29 & $05: 31: 08.7$ & $+12: 03: 46.7$ & 0.13 & No \\
\hline 4 & LB22 & $05: 31: 31.6$ & $+12: 04: 14.7$ & 0.13 & No \\
\hline 5 & LB24 & $05: 31: 23.5$ & $+12: 04: 30.7$ & 0.13 & No \\
\hline 6 & LB23 & $05: 31: 29.2$ & +12.04 .38 .7 & 0.13 & No \\
\hline 7 & LB21 & $05: 31: 36.0$ & $+12: 05: 02.7$ & 0.13 & No \\
\hline 8 & LB28 & 05:31:07.6 & $+12: 05: 06.7$ & 0.13 & No \\
\hline 9 & LB26 & $05: 31: 17.7$ & $+12: 05: 06.7$ & 0.13 & No \\
\hline 10 & LB25 & $05: 31: 20.5$ & $+12: 05: 06.7$ & 0.14 & No \\
\hline 11 & LB27 & $05: 31: 13.4$ & $+12: 05: 30.7$ & 0.13 & No \\
\hline 12 & LB20 & $05: 31: 22.9$ & $+12: 05: 30.7$ & 0.12 & No \\
\hline 13 & LB19 & $05: 31: 28.1$ & $+12: 05: 30.7$ & 0.20 & YES \\
\hline 14 & LB18 & $05: 31: 34.9$ & $+12: 06: 22.7$ & 0.12 & No \\
\hline 15 & LB17 & 05:31:09.8 & $+12: 06: 42.7$ & 0.13 & No \\
\hline 16 & LB15 & $05: 31: 27.3$ & $+12: 07: 26.7$ & 0.17 & No \\
\hline 17 & LB16 & $05: 31: 30.0$ & $+12: 07: 06.7$ & 0.16 & No \\
\hline 18 & LB14 & $05: 31: 19.4$ & $+12: 09: 10.7$ & 0.25 & YES \\
\hline 19 & LB13 & $05: 31: 18.5$ & $+12: 09: 58.7$ & 0.17 & No \\
\hline 20 & LB12 & $05: 31: 27.0$ & $+12: 10: 14.7$ & 0.17 & No \\
\hline 21 & LB11 & $05: 31: 10.4$ & $+12: 10: 38.7$ & 0.17 & No \\
\hline 22 & LB10 & $05: 31: 09.5$ & $+12: 11: 02.7$ & 0.23 & YES \\
\hline 23 & LB09 & $05: 31: 18.5$ & $+12: 11: 26.7$ & 0.17 & No \\
\hline 24 & LB08 & $05: 31: 23.2$ & $+12: 11: 26.7$ & 0.26 & YES \\
\hline 25 & LB07 & $05: 31: 32.2$ & $+12: 11: 46.7$ & 0.17 & No \\
\hline 26 & LB06 & $05: 31: 30.8$ & $+12: 12: 46.7$ & 0.17 & No \\
\hline 27 & LB05 & $05: 31: 29.5$ & $+12: 14: 30.7$ & 0.17 & No \\
\hline 28 & LB04 & $05: 31: 30.5$ & $+12: 17: 18.7$ & 0.17 & No \\
\hline 29 & LB03 & $05: 31: 29.2$ & $+12: 17: 22.7$ & 0.17 & No \\
\hline 30 & LB02 & $05: 31: 32.2$ & $+12: 19: 14.7$ & 0.17 & No \\
\hline
\end{tabular}

Notes. ${ }^{(a)}$ From BGH16; ${ }^{(b)}$ Phase center coordinates. 